\title{
SEARCH FOR DIFFERENT LINKS WITH THE SAME JONES' TYPE POLYNOMIALS: IDEAS FROM GRAPH THEORY AND STATISTICAL MECHANICS
}

\author{
JÓZEF H. PRZYTYCKI \\ Department of Mathematics, University of California \\ Berkeley, CA 94720, USA \\ e-mail: jozef@math.berkeley.edu \\ and \\ Institute of Mathematics, Warsaw University \\ Banacha 2, 02-097 Warszawa, Poland \\ e-mail: jozef@mimuw.edu.pl
}

\begin{abstract}
We describe in this talk three methods of constructing different links with the same Jones type invariant. All three can be thought as generalizations of mutation. The first combines the satellite construction with mutation. The second uses the notion of rotant, taken from the graph theory, the third, invented by Jones, transplants into knot theory the idea of the Yang-Baxter equation with the spectral parameter (idea employed by Baxter in the theory of solvable models in statistical mechanics). We extend the Jones result and relate it to Traczyk's work on rotors of links. We also show further applications of the Jones idea, e.g. to 3-string links in the solid torus. We stress the fact that ideas coming from various areas of mathematics (and theoretical physics) has been fruitfully used in knot theory, and vice versa.
\end{abstract}

0. Introduction. Exactly ten year ago, at spring of 1984, Vaughan Jones introduced his (Laurent) polynomial invariant of links, $V_{L}(t)$. He checked immediately that it distinguishes many knots which were not taken apart by the Alexander polynomial, e.g. the right handed trefoil knot from the left handed trefoil knot, and the square knot from the granny knot; Fig. 0.1.

1991 Mathematics Subject Classification: Primary: 57M25. Secondary: 82B.

Key words: knot, link, Jones polynomial, skein polynomial, transfer matrices, Yang-Baxter equation.

This is the detailed version of the talk given at the Banach Center Colloquium on 24th March 1994 ("W poszukiwaniu nietrywialnego wȩzła z trywialnym wielomianem Jonesa: grafy i mechanika statystyczna").

The paper is in final form and no version of it will be published elsewhere. 

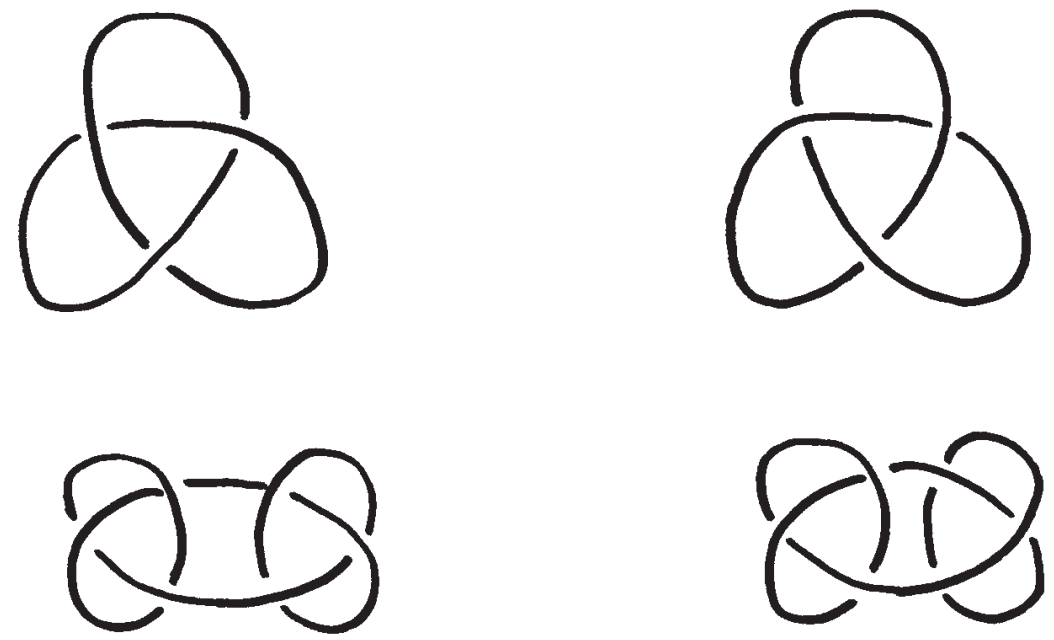

Fig. 0.1

Jones also noticed that his polynomial is not universal. That is, there are different knots with the same polynomial; e.g. the Conway and Kinoshita-Terasaka knots; Fig. 0.2.
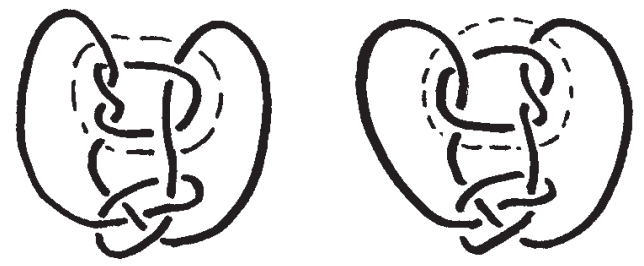

Fig. 0.2

Then Jones asked the fundamental question whether there exists a nontrivial knot with the trivial polynomial. Ten years later this is still an open problem and specialists differ in their opinion whether the answer is yes or no. In this talk, I will concentrate on more accesible problem: how to construct different links with the same Jones polynomial. It may shed some light into the Jones question. I will describe three methods of constructing knots with a coinciding Jones polynomial (and its generalizations), each of which can be thought as a generalization of the Conway idea of mutation. First however, in the introduction, we remind the definitions of Jones type polynomials and of the Conway's mutation.

(1) In the first part we consider satellites of mutants and their Jones type invariants.

(2) In the second part we explain the idea of rotors.

(3) In the third part we explore the idea of Jones of the spectral parameter tangle. 
(4) In the fourth part we apply the idea to the skein polynomial of links.

(5) In the fifth part we use a 3-string spectral parameter tangle.

We remind now definitions of the Jones polynomial, $V_{L}(t)$, and its generalizations: the skein (Homflypt) polynomial, $P_{L}(v, z)$, and Kauffman polynomial, $F_{L}(a, x)$

Definition 0.1. The skein polynomial invariant of oriented links can be characterized by the recursive relation (skein relation):

(i) $v^{-1} P_{L_{+}}(v, z)-v P_{L_{-}}(v, z)=z P_{L_{0}}(v, z)$, where $L_{+}, L_{-}$and $L_{0}$ are three oriented link diagrams, which are the same outside a small disk in which they look as in Fig. 0.3,

and the initial condition

(ii) $P_{T_{1}}=1$, where $T_{1}$ denotes the trivial knot.

The Jones polynomial is defined as $V_{L}(t)=P_{L}\left(t, \sqrt{t}-\frac{1}{\sqrt{t}}\right)$. The Alexander polynomial, $\Delta_{L}(t)$, as normalized by Conway, satisfies $\Delta_{L}(t)=P_{L}\left(1, \sqrt{t}-\frac{1}{\sqrt{t}}\right)$.

Kauffman $[20]$ gave different approach to the Jones polynomial, starting from the invariant of regular isotopy of unoriented diagrams or, equivalently, working with unoriented framed links. This variant of the Jones polynomial is called now the Kauffman bracket polynomial.

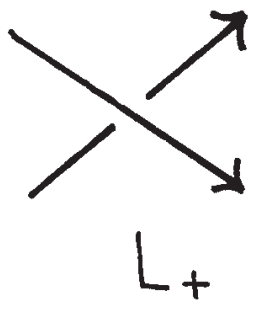

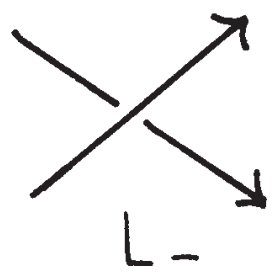

Fig. 0.3

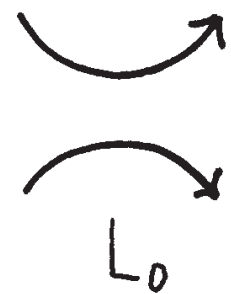

Definition 0.2. The Kauffman bracket polynomial, $\langle L\rangle \in Z\left[A^{ \pm 1}\right]$, of framed unoriented links is characterized by the recursive relation (Kauffman bracket skein relation):

(i) $\left\langle L_{A}\right\rangle=A\left\langle L_{0}\right\rangle+A^{-1}\left\langle L_{\infty}\right\rangle$, where $L_{A}, L_{0}$ and $L_{\infty}$ denote diagrams of unoriented framed links, which are the same outside a small disc in which they look as in Fig. 0.4. We use the convention that the framing of the diagram is vertical to the plane of projection, unless otherwise stated.

(ii) Initial conditions:

$\left\langle T_{n}\right\rangle=\left(-A^{2}-A^{-2}\right)^{n-1}$, where $T_{n}$ is the trivial framed $n$-component link.

If we orient $L$, then we get the Jones polynomial

$$
V_{L}\left(A^{-4}\right)=\left(-A^{3}\right)^{-\operatorname{Tait}(L)}\langle L\rangle,
$$


where Tait $(L)$ is the sum of signs of crossings of $L$. Equivalently, for a framed oriented link $L$, Tait $(L)$ is "the defect" of the framing, that is the number of negative twists minus the number of positive twists which have to be performed on the framing of $L$ so that the new framing agrees with that given by the Seifert surface of $L$. Let us use the convention that $L^{(1)}$ denote a framed link obtained from $L$ by twisting the framing of $L$ once in the positive (right handed) direction. Notice that $L^{(1)}$ is not uniquely defined if $L$ is not a knot, but its Kauffman bracket is well defined and the condition (ii) can be replaced by:

(iii) $\left\langle L^{(1)}\right\rangle=-A^{3}\langle L\rangle$.

To introduce the Kauffman polynomial, it is, as before, very convenient to define it first for unoriented framed links.

Definition 0.3. The Kauffman polynomial of framed unoriented links, $\Lambda_{L}(a, x) \in Z\left[a^{ \pm 1}, x^{ \pm 1}\right]$, is characterized by the recursive relation (Kauffman skein relation):

(i) $\Lambda_{L_{A}}(a, x)+\Lambda_{L_{B}}(a, x)=x\left(\Lambda_{L_{0}}(a, x)+\Lambda_{L_{\infty}}(a, x)\right)$, where $L_{A}, L_{B}, L_{0}$ and $L_{\infty}$ are four diagrams of unoriented framed links, which are the same outside a small disc in which they look as in Fig. 0.4.

(ii) Framing relations:

$L^{(1)}=a L$.

(iii) Initial condition:

$\Lambda_{T_{1}}=1$, for the trivial framed $\operatorname{knot} T_{1}$.

If we orient $L$, then the Kauffman polynomial of the oriented unframed link $L, F_{L}(a, x)$, is defined to be: $F_{L}(a, x)=a^{-\operatorname{Tait}(L)} \Lambda_{L}(a, x)$.

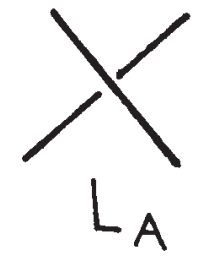

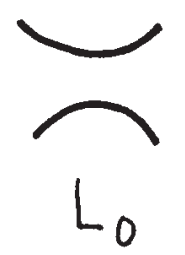

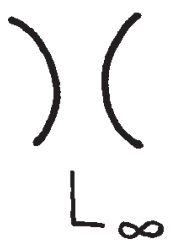

Fig. 0.4

The simplest method of producing different links with the same invariant is mutation invented before 1960 by J. Conway. We describe the Conway idea of tangles and mutation below ${ }^{1}$ :

Consider a 2-tangle, $L$, that is a part of a link diagram placed in a disk, with 4 boundary points ( 2 inputs and 2 outputs, if $L$ is oriented); see Fig. 0.5.

\footnotetext{
${ }^{1}$ Conway recalls working out a good part of his theory of tangles while still a high school student $[1]$.
} 


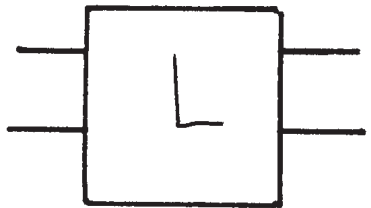

(a)

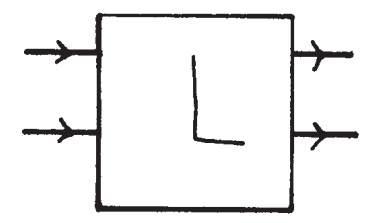

(b)

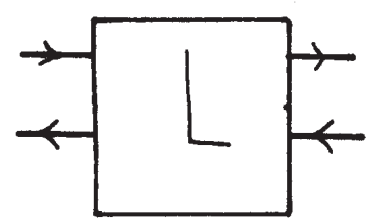

(c)

Fig. 0.5

We perform a mutation of the link, of which $L$ is a part, by rotating the tangle along the $x, y$ or $z$ coordinate axis by the angle $\pi$. Thus we have three mutations $m_{x}, m_{y}$ and $m_{z}$, respectively; Fig. 0.6. Notice that together with the identity map they form the group $D_{2}=Z_{2} \oplus Z_{2}$. We keep the part of the link outside the tangle fixed and, if necessary, change the orientation of the tangle part so it agrees with outside part of the link.

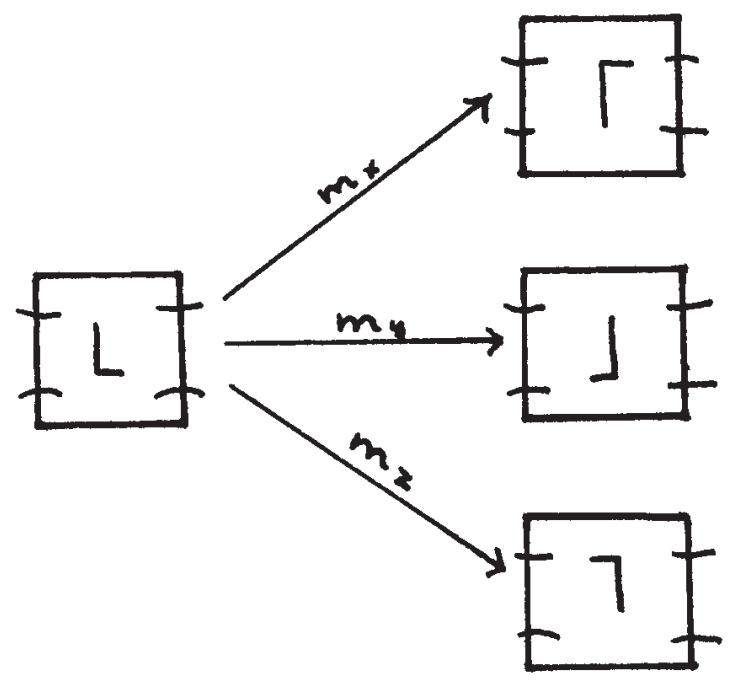

Fig. 0.6

The mutation preserves not only Jones type polynomials but also the volume of the (hyperbolic) complement of a link and the homeomorphic type of the branched double cover of $S^{3}$ with the link as the branching set, among many
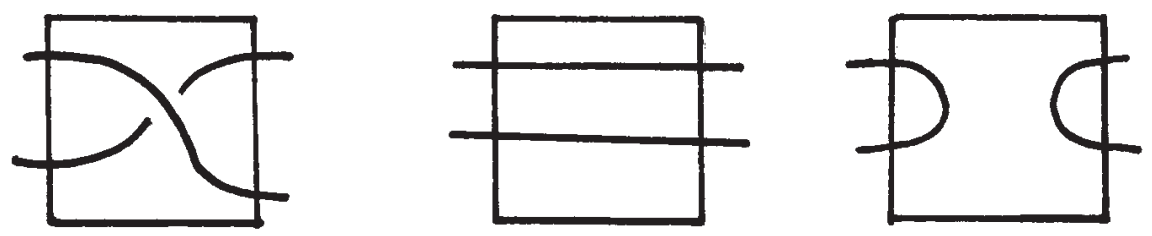

Fig. 0.7 
other invariants. The simplest pair of non-equivalent mutant knots, the Conway and Kinoshita-Terasaki knots, are drawn in Fig. 0.2.

To show that Jones type invariants are preserved by mutation, one follows Conway's idea of skein theory [6, 24, 8]. Namely, one simplifies the 2-tangle of mutation as far as possible reaching, finally, tangles which are invariant under mutation. In the case of the Kauffman skein relation one gets one of the tangles of Fig. 0.7 with possible additional trivial components. Of course, each of these tangles is invariant under any mutation.

1. Satellites of mutants. One can produce more complicated links from the given one, say $L$, by decorating each component of the link by some pattern. The resulting link is called a satellite of $L$. If we consider satellites of mutants (with the same pattern) we obtain links with the same Jones polynomial and, sometimes, with the same skein and Kauffman polynomials.

We should stress, however, that we cannot produce, in such a way, a nontrivial knot with a polynomial of the trivial knot. It is the case because a mutation of a trivial knot is a trivial knot (the 2-fold branched cover of $\left(S^{3}, L\right)$ and $\left(S^{3}, m(L)\right)$ are homeomorphic [25, 44], and the 2-fold branched cover of $S^{3}$ whith a nontrivial knot as the branching set cannot be $S^{3}$; [45]). Furthermore a nontrivial satellite of a nontrivial knot is a nontrivial knot.

TheOREM $1.1[26]$. Let $D$ be a diagram of a framed unoriented link and let $m(D)$ be a mutant of $D$. Assume additionally that the mutation preserves link components of $D$ (i.e. if $v$ is a boundary point of the rotated tangle, then $v$ and $m(v)$ lie in the same link component of $D)$. Then for any satellites of $D$ and $m(D)$ with the same pattern $s$ (denoted by $s(D)$ and $s(m(D))$, respectively), the Kauffman bracket polynomial is the same. That is $\langle s(D)\rangle=\langle s(m(D))\rangle$. If $D$ is additionally oriented then $V_{s(D)}(t)=V_{s(m(D))}(t)$.

Theorem $1.2[23,29]$. Let $m(D)$ be a mutant of $D$ obtained by rotating a tangle $T$ and preserving link components of $D$. Let $s$ be a satellite pattern with the wrapping number at most 2 (e.g. 2-cable or Whitehead double). Then:

(a) If $D$ is oriented then:

(i) $P_{s(D)}(v, z)=P_{s(m(D))}(v, z)$,

(ii) $F_{s(D)}(a, x)=F_{s(m(D))}(a, x)$.

(b) If $D$ is an unoriented framed diagram, then $\Lambda_{s(D)}(a, x)=\Lambda_{s(m(D))}(a, x)$.

Theorem 1.2 does not hold for wrapping number greater than 2. For example, Morton and Traczyk [26] and J. Murakami [28] have shown that a 3-cable of the Conway knot and its mutant the Kinoshita-Terasaka knot have different skein and Kauffman polynomials. However a weaker fact still holds: 
Theorem $1.3[21,29]$. Let $L_{1}$ and $L_{2}$ be two oriented links and let $-L_{2}$ be obtained from $L_{2}$ by reversing orientations of all components of $L_{2}$. Consider connected sums $L_{1} \# L_{2}$ and $L_{1} \#-L_{2}$, where the same components of $L_{1}$ and $L_{2}$ are involved in $L_{1} \# L_{2}$ and $L_{1} \#-L_{2}$ (the second sum can be thought as a degenerated, components preserving, mutation of the first). Then for any pattern $s$, the satellites $s\left(L_{1} \# L_{2}\right)$ and $s\left(L_{1} \#-L_{2}\right)$ have the same skein and Kauffman polynomials.

The original proofs of Theorems $1.1-1.3$ were combinatorial, using skein theory similarly as in the proof of the case of mutation. Later, however, J. Murakami and G. Kuperberg found proofs based on properties of irreducible representations of Lie algebras.

Theorems 1.1 and 1.3 are the basic tools in constructing different 3-manifolds with the same Witten invariants [19, 22]. Theorem 1.1 is used in the case of $S U(2)$ Witten invariant, as constructed by Reshetikhin and Turaev [35] and Theorem 1.3 in the case of classical Lie algebra Witten invariants, as constructed by Turaev and Wenzl [42].

2. Rotors. The idea of rotors was used first in graph theory in the fundamental paper on division of a square into smaller unequal squares [5]. Later Tutte used it to produce different graphs with the same dichromatic polynomial [43]. The idea was "translated" into knot theory in the paper of R. Anstee, D. Rolfsen and myself.

Definition 2.1 [2]. Consider an $n$-tangle, that is a part of the link diagram (possibly oriented or framed - depending on the application), placed in the regular $n$-gon with $2 n$ boundary points ( $n$ inputs and $n$ outputs). We say that this $n$-tangle is an $n$-rotor if it has a rotational symmetry, that is the tangle is invariant with respect to rotation along $z$-axis by the angle $\frac{2 \pi}{n}$; see Fig. 2.1 for an example of a 4-rotor.

THEOREM 2.2 [2]. Let $L$ be a link diagram with an $n$-rotor part $R$. Let the rotant $r(L)$, be obtained from $L$ by rotating $R$ along the $x$-axis by the angle $\pi$ and keeping the stator, $S=L-R$, unchanged (if necessary, we change the orientation of the rotor so it agrees with that of the stator). Then:

(a) $\langle L\rangle=\langle r(L)\rangle$ for $n \leq 5$, where $L$ is an unoriented framed link diagram.

(b) $P_{L}(v, z)=P_{r(L)}(v, z)$ for $n \leq 4$, where $L$ is an oriented link diagram.

(c) $\Lambda_{L}(v, x)=\Lambda_{r(L)}(v, x)$ for $n \leq 3$, where $L$ is an unoriented framed link diagram.

Furthermore, if $L$ is oriented then $\operatorname{Tait}(L)=\operatorname{Tait}(r(L))$.

The proof of the theorem given in [2] is a straightforward generalization of the proof for mutants: the skein relation allows as to simplify the stator of $L$ so that it is invariant under the reflection in a side of the $n$-gon. We illustrate it 


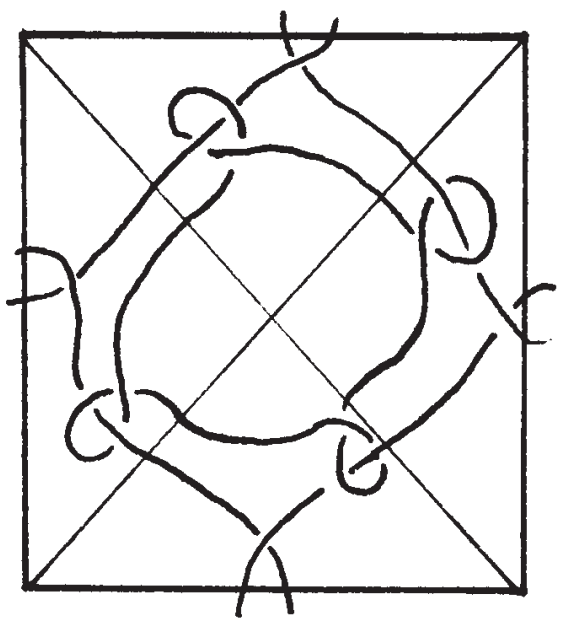

Fig. 2.1. Rotor of Toulouse, Carolingian era, IX century AD.

for $n=3$ and the Kauffman skein relation. If we simplify the stator (placed in a regular triangle) using the Kauffman skein relations, we obtain one of the fifteen 3 -tangles (possibly with additional trivial components); Fig. 2.2.
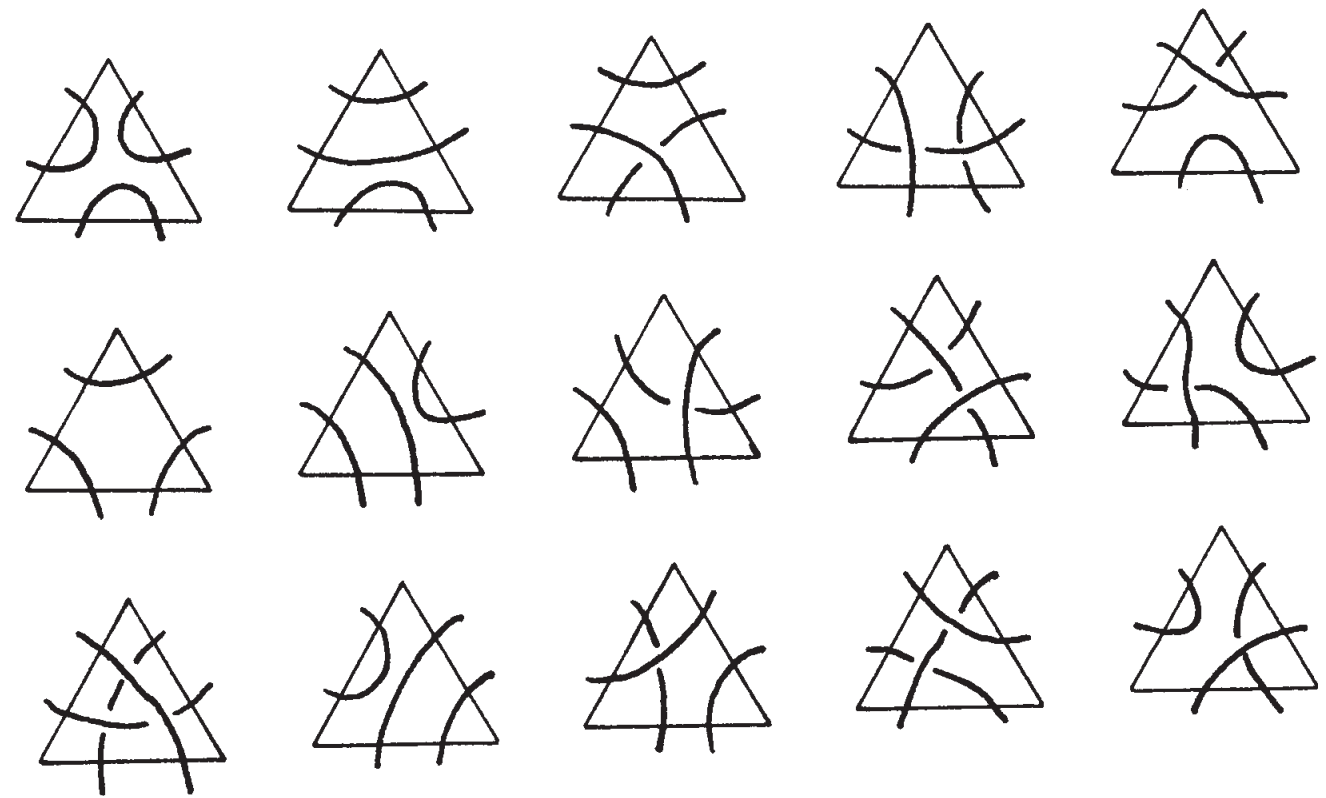

Fig. 2.2

Clearly each tangle of Fig. 2.2 is symmetric with respect to reflection in an edge of the triangle. Instead of reflecting the stator we can reflect the rotor (and because of its rotational symmetry, all reflections are equivalent), and the Theorem 2.2(c) follows. 
D. Rolfsen was searching for examples of $n$-rotors for which the theorem does not hold. In particular he studied 6-rotors and their Jones polynomial. However he couldn't find a counterexample to the theorem. At the Sussex conference held in summer of 1987, he discussed rotors with P. Traczyk and Traczyk observed that 6-rotors considered by Rolfsen have only 2 connections between identical segments of rotors; see Fig. 2.3.

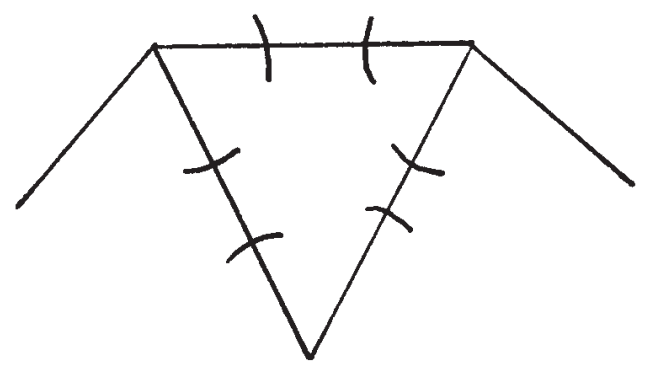

Fig. 2.3

T. Jin and Rolfsen considered more complicated rotors and found, in the summer of 1988, that Theorem 2.2 is the best possible [11].

On the other hand, Traczyk showed that if there are only two internal connections between segment of a rotor then the Jones and the skein polynomials are the same for the link and its rotant (he proved it first for $n=6$ in 1987, and the next year in the full generality). Namely:

Theorem 2.3 [39]. Let $L$ be a link diagram with an n-rotor part $R$. Furthermore assume that there are at most two arcs between neighboring segments of the rotor. If $r(L)$ is the rotant of $L$ then:

(a) $\langle L\rangle=\langle r(L)\rangle$, for any $n$, where $L$ is an unoriented framed link diagram.

(b) $P_{L}(v, z)=P_{r(L)}(v, z)$, for any $n$, where $L$ is an oriented link and a segment of the rotor (which is a 3-tangle) is oriented, up to the global change of orientation, as in Fig. 2.4.

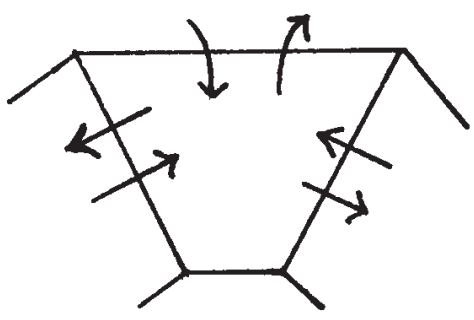

Fig. 2.4

The method used by Traczyk for proving Theorem 2.3 is essentially different than that used in the proof of Theorem 2.2. Namely, it is algebraic and uses essentially the linearity of skein relations. Furthermore Traczyk operates on the 
rotor part of the link (excluding its center) instead of the stator, so he gets, in fact, theorem about tangles in the solid torus (projected into an annulus), or any 3 -manifold in which the solid torus is embedded (to make it precise one should consider the notion of the skein module of a 3-manifold; see part 3). Traczyk's theorem does not hold for the Kauffman polynomial. A simple counterexample is given in [11] (Example 1); see Fig. 2.5, where 4-rotors with different Kauffman polynomials are presented. Furthermore these rotors have only two connections between segments.
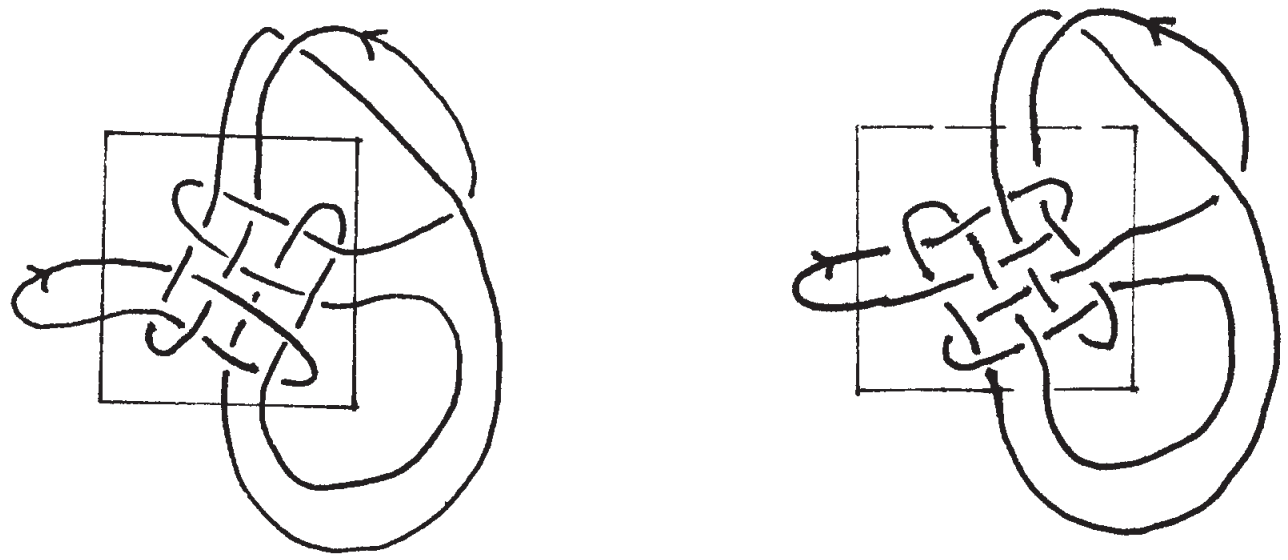

Fig. 2.5. $F_{L}(a, x)-F_{r(L)}(a, x)=\left(a^{10}+4 a^{12}+6 a^{14}+4 a^{16}+a^{18}\right)+\mathcal{O}(z)$.

It follows from the work of Tutte that the determinant of an oriented link, $\Delta_{L}(-1)$, is the same for $L$ and its rotant $r(L)$, for any $n$ and any number of connection between segments. This suggests the possibility that the whole Alexander polynomial is unchanged by rotation and a lot of examples which confirm this were computed by Jin and Rolfsen.

Conjecture 2.4. If $L$ is an oriented link and $r(L)$ its rotant then their Alexander polynomials are equal.

We noticed in [2] that if we decorate an unoriented framed link $L$ and its 2-rotant $r(L)$ by a pattern $s$ with the wrapping number 2, then the Kauffman bracket polynomials of $s(L)$ and $s(r(L))$ are equal. There is no need, however, for a separate proof of this fact because Yamada showed [46] that the Kauffman bracket of a 2-cable of a link is determined by the Kauffman polynomial of the link at $(a, x)=\left(i A^{8},-i\left(A^{4}-A^{-4}\right)\right)$. The idea of rotors was taken from graph theory ${ }^{2}$. Now however, Traczyk's theorem and the results of the next parts can be translated back to give new results in graph theory.

\footnotetext{
2 One can say that it came from physics, as the authors of [5] where motivated by the theory of electrical circuits.
} 
3. The spectral parameter tangle. In the spring of 1991, Jones used an idea from the statistical mechanics to produce examples of different links with the same Jones type polynomials. We describe in this part of the talk how the YangBaxter equation with spectral parameter can be "translated" into an equation involving tangles and how the Baxter method of "commuting transfer matrices" can be "translated" to produce various links with the same Jones, and skein polynomials $[14,15]$. After this we generalize slightly the method of Jones, and relate it to Traczyk's work on rotors of links ${ }^{3}$. Finally we give another application of the spectral parameter tangles.

We first present the work of Jones.

Consider a finite dimensional vector space $V$ and the space of parameters $\Lambda$. Consider also the family, $R(\lambda)$, of matrices $(R: \Lambda \rightarrow \operatorname{End}(V \otimes V))$. We say that $R$ satisfies the Yang-Baxter equation with spectral parameter if for every pair $\lambda, \lambda^{\prime} \in \Lambda$ there is $\lambda^{\prime \prime} \in \Lambda$ such that

$$
R_{1}(\lambda) R_{2}\left(\lambda^{\prime}\right) R_{1}\left(\lambda^{\prime \prime}\right)=R_{2}\left(\lambda^{\prime \prime}\right) R_{1}\left(\lambda^{\prime}\right) R_{2}(\lambda),
$$

where both sides are endomorphisms of $V \otimes V \otimes V$ and $R_{1}(\chi)=R(\chi) \otimes \operatorname{Id}$ and $R_{2}(\chi)=\operatorname{Id} \otimes R(\chi) . \quad \chi \in \Lambda$ is called the spectral parameter. The Yang-Baxter equation is expressed graphically in Figure 3.1.
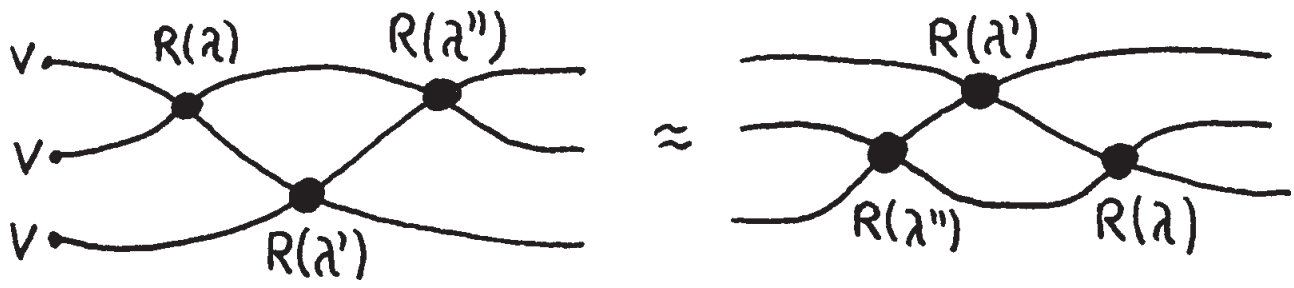

Fig. 3.1

We interpret three initial points as $V \otimes V \otimes V$ and going through the crossing corresponds to the endomorphism $R(\chi)$. When the spectral parameter is constant then the correspondence between the crossing and the Yang-Baxter operator $R$ can be used to define new, Jones type, invariants of links (Jones [13], Turaev [40]). The method uses the fact that a Yang-Baxter equation corresponds to the third Reidemeister move (Fig. 3.2).

V. Jones in the spring of $1991[14,15]$ discovered that one can use the idea of the Yang-Baxter operator with spectral parameter to produce different links with the same Jones polynomials. The idea uses the classical argument from the theory of solvable models in statistical mechanics [3]. As before, $V$ corresponds to a point and $\underbrace{V \otimes V \otimes \ldots \otimes V}_{n}$ to $n$ points. $R(\lambda) \in \operatorname{End}(V \otimes V)$ corresponds to a

\footnotetext{
3 This part and Part 4 are based on the notes for the talk given at the University of Tennessee, October 1991 [31].
} 

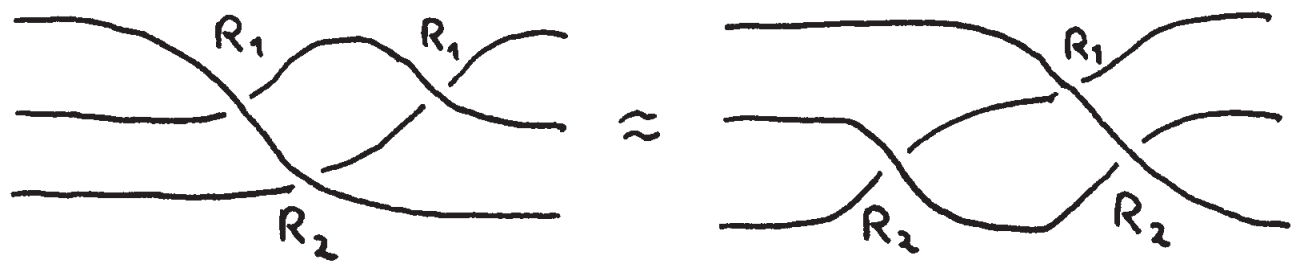

Fig. 3.2

2-tangle $L$ and the composition of endomorphisms corresponds to the composition of tangles. To get the analogy to the Yang-Baxter equation we consider the skein module (linear skein) of a tangle, the notion which was essentially introduced by J. Conway $([6,9,30,41])$. In short skein modules are quotients of modules over ambient isotopy classes of links in a 3-manifold (possibly with boundary) by properly chosen local (skein) relations.

Now we have to be more specific and choose a skein module with which we work. We start with the Kauffman bracket approach to the Jones polynomial. Consider an $n$-tangle, that is a 2 -disc with fixed $2 n$ points on the boundary and a framed link diagram (composed of closed curves and curves with endpoints fixed) inside. Framing is fixed at boundary points. The Kauffman bracket skein module of $n$-tangles, $\mathcal{S}_{2, \infty}(n)$ is defined to be an $R$-module (for a chosen commutative ring with 1 ) obtained from the free $R$-module over all tangles up to isotopy (modulo boundary) divided by the submodule generated by (the Kauffman bracket) skein relations $L_{A}=A L_{0}+A^{-1} L_{\infty}$ and framing relations $L^{(1)}=-A^{3} L$, where $L^{(1)}$ denotes a framed link obtained from $L$ by twisting its framing once in the positive direction. $A$ is an invertible element of $R$ (in practice, unless otherwise stated, we will assume at this talk that $R=\mathcal{F}(A)$ - the field of rational functions in variable $A) \cdot \mathcal{S}_{2, \infty}(n)$ is a free module of $\frac{1}{n+1}\left(\begin{array}{c}2 n \\ n\end{array}\right)$ generators ([12, 27]). For example $\mathcal{S}_{2, \infty}(2)$ has two generators and $\mathcal{S}_{2, \infty}(3)$ has five generators; Fig. 3.3.
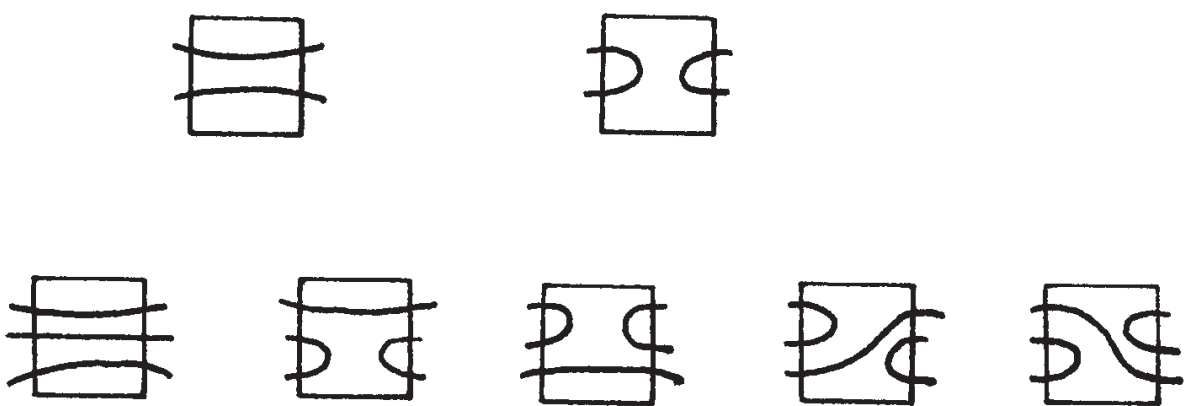

Fig. 3.3 
$\mathcal{S}_{2, \infty}(n)$ with product yielded by the composition of tangles has a structure of an algebra (the Temperley-Lieb algebra [38]). We will use the standard (in Yang-Baxter equations theory) notation: $R_{(i)}$ for a 2-tangle $R$ placed on $i$ th and $(i+1)$ 'th strings of $n$ strings, see Fig 3.4. The result (analogy to the YangBaxter equation with spectral parameter) which Jones uses, can be stated as follows:

Lemma 3.1 (Jones [15]). For a dense subset of pairs of tangles $T, T^{\prime} \in \mathcal{S}_{2, \infty}(2) \times$ $\mathcal{S}_{2, \infty}(2)$ there is an invertible tangle $T^{\prime \prime}$ (in Temperley Lieb algebra $\mathcal{S}_{2, \infty}(2)$ ) such that: $T_{(1)} T_{(2)}^{\prime} T_{(1)}^{\prime \prime}=T_{(2)}^{\prime \prime} T_{(1)}^{\prime} T_{(2)}$. Graphically this equality of two 3-tangles is shown in Figure 3.4.
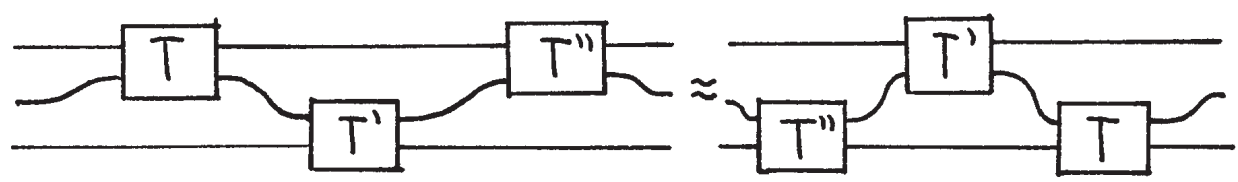

\section{or equivalently}
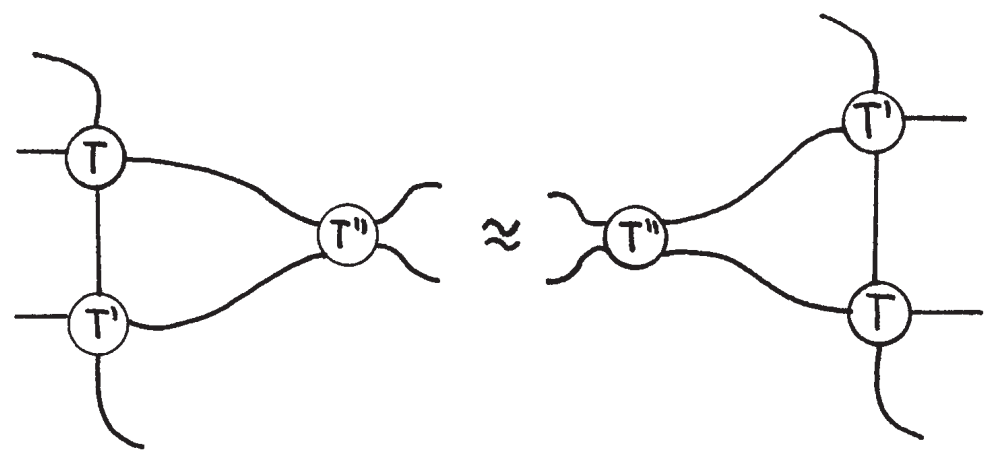

Fig. 3.4

We do not prove here the Jones lemma as its generalized version is given in Lemma 3.3. The "trick" which Jones uses goes as follows:

Consider 2-tangles $T$ and $T^{\prime}$ placed cyclicly in the annulus as in Figure 3.5(a). Assume that for $T$ and $T^{\prime}$, there exists an invertible 2-tangle $T^{\prime \prime}$ from Lemma 3.1. Then without changing the element of the Kauffman bracket skein module of the solid torus (annulus is the projection surface of the solid torus), we can insert the pair of tangles $T^{\prime \prime}$ and $\left(T^{\prime \prime}\right)^{-1}$ (Fig. 3.5(b)) and then move around the annulus with $T^{\prime \prime}$, interchanging $T$ with $T^{\prime}$ on the way (Fig. 3.5(c)), and finally arriving on the second side of $\left(T^{\prime \prime}\right)^{-1}$ and canceling it; Fig. 3.5(d). 

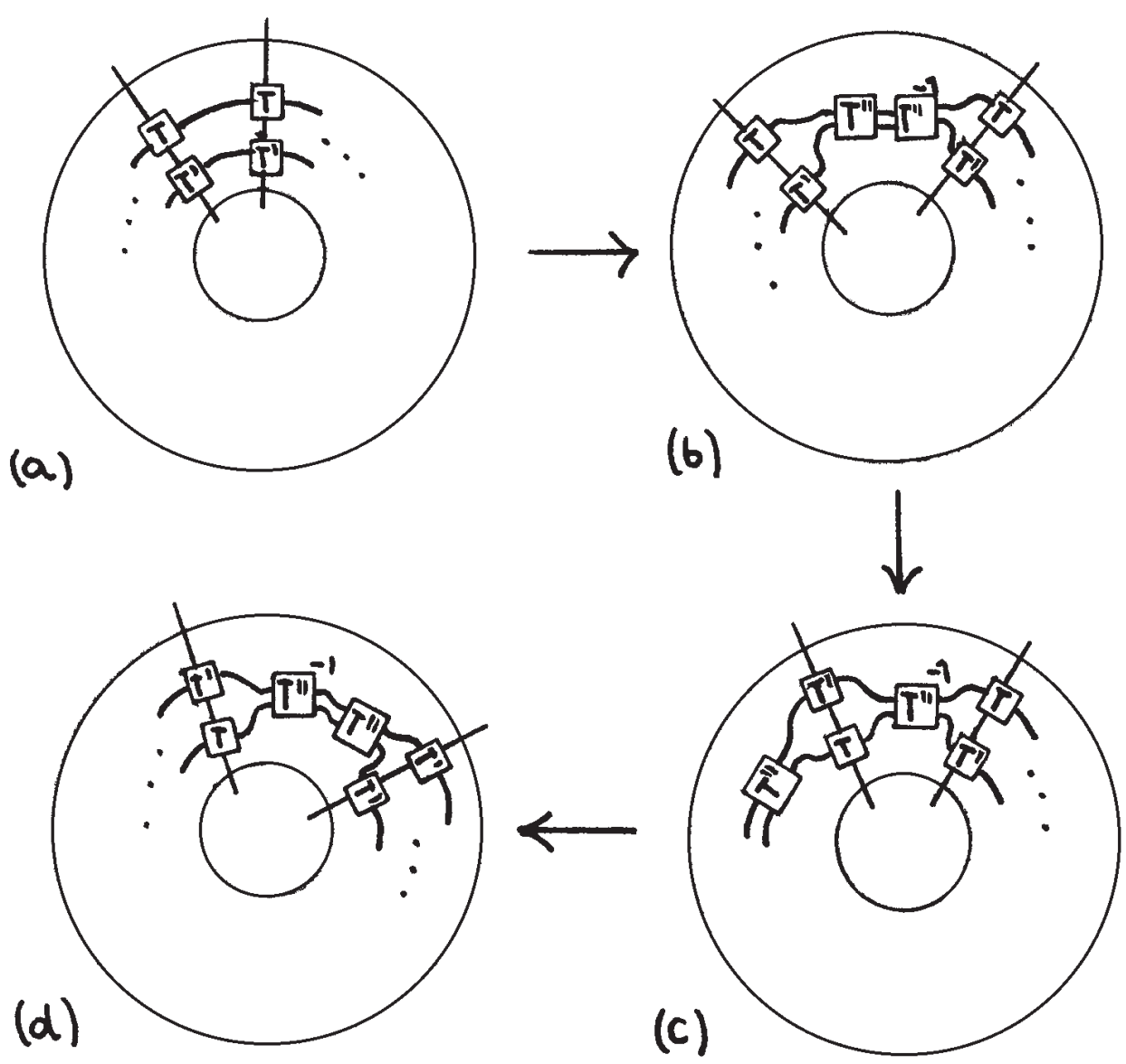

Fig. 3.5

One sligtly extends the scope of the Jones "trick" by observing that $\mathcal{S}_{2, \infty}(2)$ is commutative. Thus one gets

Theorem 3.2 [15]. Let $T$ and $T^{\prime}$ be 3 -tangles and $s_{i}, i=1, \ldots, n$, 2-tangles. Consider the following elements of the Kauffman bracket skein module of the solid torus with $2 n$ boundary points; Fig. 3.6(a) and (b).

Then the tangles (a) and (b) represent the same element of the Kauffman bracket skein module of the solid torus. In particular, if the solid torus is embedded in an oriented 3-manifold and boundary points are connected together (outside the solid torus), then the links obtained are equal in the Kauffman bracket skein module of the 3-manifold.

At the conference in Sacramento at April 1991, Jones gave a talk explaining his ideas. We discussed them also afterwards (Jones, Hoste and myself). I knew well the work of Traczyk on rotors so I suspected immediately that it should be related to results of Jones. In fact I noticed that the Jones method works without 
(a)
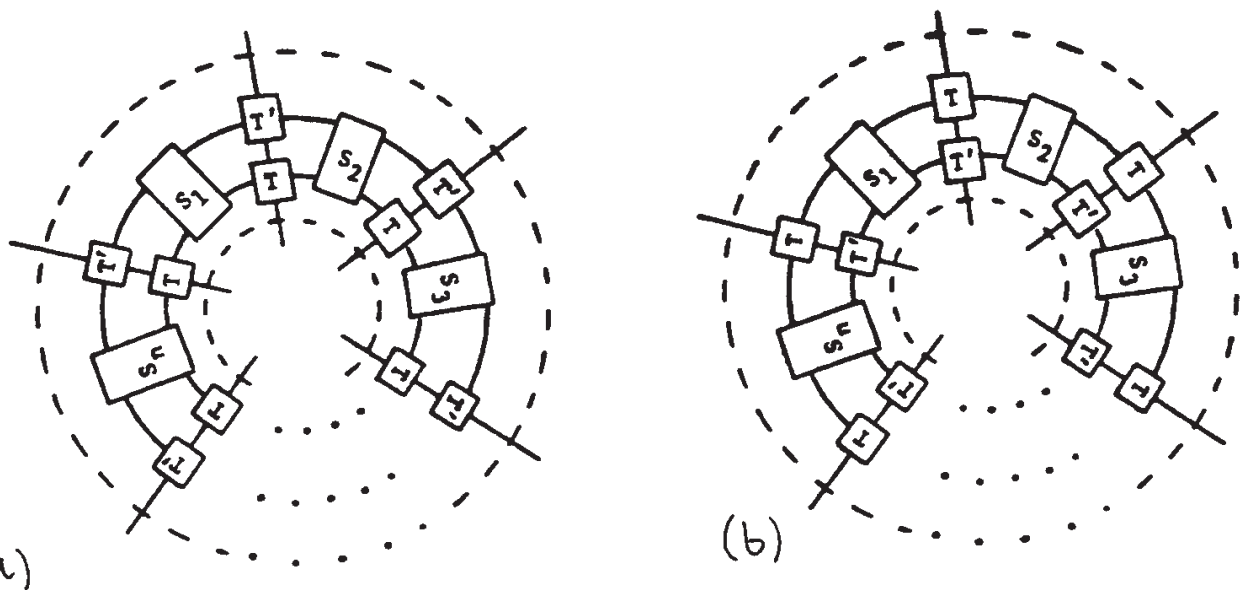

Fig. 3.6

changes if instead of two tangles $T$ and $T^{\prime}$ combined as in Figure 3.7(a), one can, more generally, consider one tangle of Figure 3.7(b). Similarly one can work with the tangle of Figure 3.7(c). In such a way one recovers the Traczyk result and generalize it. We present now, with details, this slight generalization of the Jones work. We start from the case of the Kauffman bracket polynomial (and the Kauffman bracket skein module).

(a)

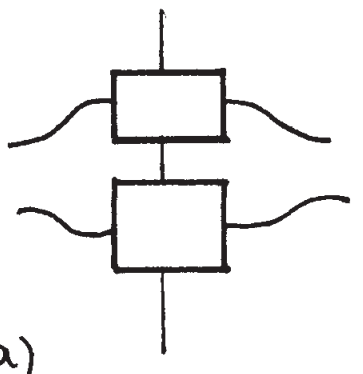

(b)

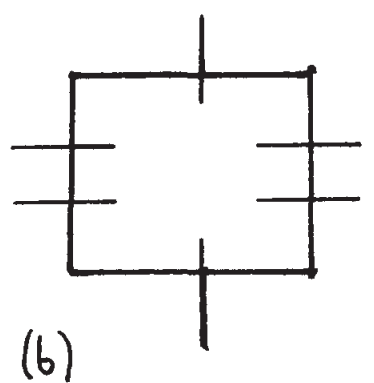

Fig. 3.7

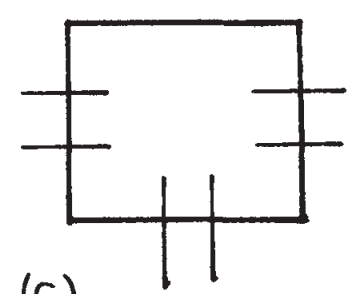

(c)

LEMMA 3.3.

(a) Let $r_{z}: \mathcal{S}_{2, \infty}(n) \rightarrow \mathcal{S}_{2, \infty}(n)$ denote the algebra isomorphism generated by rotating a tangle by 180 degrees about the z-axis $\left(r_{z}(L)=7\right)$. Then for a dense subset of tangles $L \in \mathcal{S}_{2, \infty}(3)$ there is an invertible 2 -tangle $P=P(L)$ such that $L P_{(1)}=P_{(2)} r_{z}(L)$ in $\mathcal{S}_{2, \infty}(3)$ (see Figure 3.8 ).

(b) Let $r_{y}: \mathcal{S}_{2, \infty}(n) \rightarrow \mathcal{S}_{2, \infty}(n)$ denote the involution of modules given by the rotation $\left.r_{y}\left(r_{y}(L)=\right\rfloor\right)$. Then there is a dense subset $D(3)$ of elements of $\mathcal{S}_{2, \infty}(3)$, such that if $L$ is in this subset, then there exists an invertible 2-tangle $P=P(L)$ such that $L P_{(1)}=P_{(1)} r_{y}(L)$ in $\mathcal{S}_{2, \infty}(3)$ (see Figure 3.9 ). 


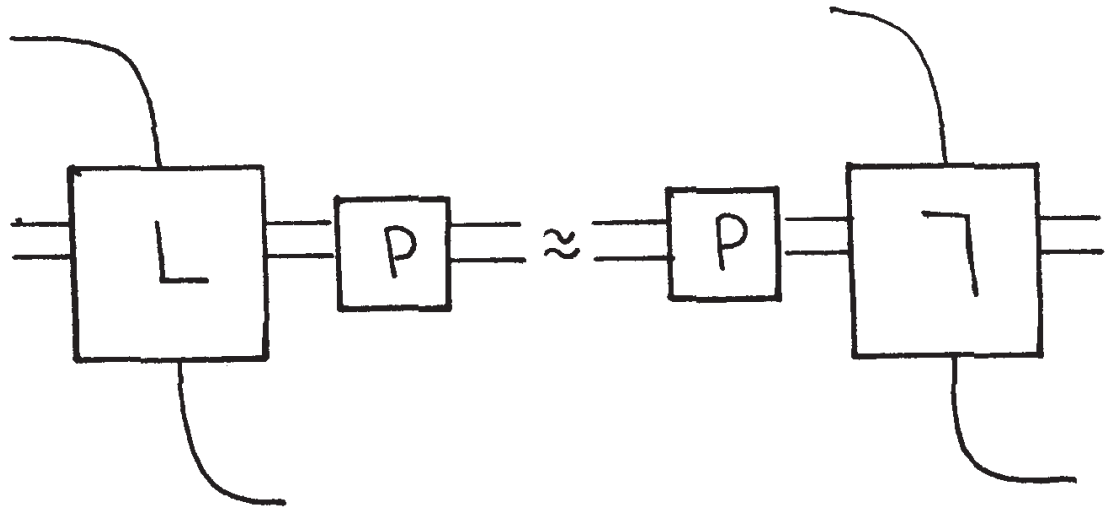

Fig. 3.8
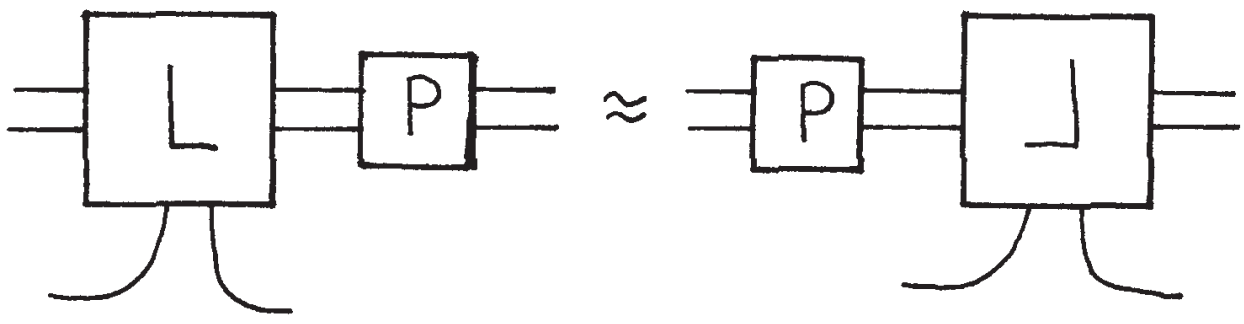

Fig. 3.9

Proof. We will prove (b) in details. The proof of (a) is similar ${ }^{4}$. Let $\left(e_{1}, e_{2}, \ldots, e_{5}\right)$ be the basis of $\mathcal{S}_{2, \infty}(3)$ and $\left(f_{1}, f_{2}\right)$ the basis of $\mathcal{S}_{2, \infty}(2)$ as shown in Figure 3.10.
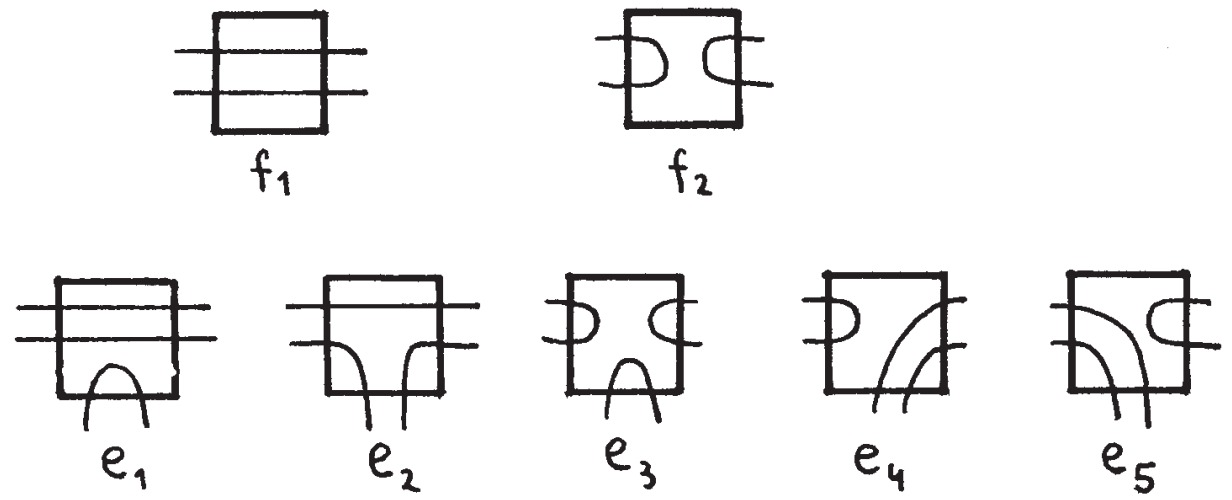

Fig. 3.10

\footnotetext{
${ }^{4}$ It is observed in [10] that (a) follows immediately from (b), thus there is no need for a separate calculation.
} 
$\mathcal{S}_{2, \infty}(3)$ is a left and a right $\mathcal{S}_{2, \infty}(2)$ module. The table for the right multiplication is shown in Figure 3.11, where $\mu=-A^{2}-A^{-2}$.

$$
\left[\begin{array}{cc}
e_{1} & e_{3} \\
e_{2} & e_{5} \\
e_{3} & \mu e_{3} \\
e_{4} & e_{3} \\
e_{5} & \mu e_{5}
\end{array}\right]
$$

Fig. 3.11. The $(i, j)$ entry of the matrix is equal to $\left(e_{i} f_{j}\right)$.

Now let $L \in \mathcal{S}_{2, \infty}(3)$ and $L=a_{1} e_{1}+a_{2} e_{2}+a_{3} e_{3}+a_{4} e_{4}+a_{5} e_{5}$. We look for $P=\left(x f_{1}+y f_{2}\right) \in \mathcal{S}_{2, \infty}(2)$, such that

$$
L P=r_{y}(L P) .
$$

$L P=\left(a_{1} x\right) e_{1}+\left(a_{2} x\right) e_{2}+\left(a_{1} y+a_{3} x+\mu a_{3} y+a_{4} y\right) e_{3}+\left(a_{4} x\right) e_{4}+\left(a_{2} y+a_{5} x+\mu a_{5} y\right) e_{5}$. Notice that $r_{y}\left(f_{i}\right)=f_{i}$ and $r_{y}\left(e_{i}\right)=e_{i}$ for $i \leq 3$, and $r_{y}\left(e_{4}\right)=e_{5}, r_{y}\left(e_{5}\right)=e_{4}$. Therefore $(* *)$ is equivalent to: $a_{4} x=a_{5} x+a_{2} y+\mu a_{5} y$ or equivalently $y\left(a_{2}+\right.$ $\left.\mu a_{5}\right)=x\left(a_{4}-a_{5}\right)$. Now, either

(i) $a_{2}+\mu a_{5}=0$ and then $x\left(a_{4}-a_{5}\right)=0$,

or

(ii) $a_{2}+\mu a_{5} \neq 0$ and then $\frac{y}{x}=\frac{a_{4}-a_{5}}{a_{2}+\mu a_{5}}$. Equivalently one has one projective solution $(x, y)=t\left(a_{2}+\mu a_{5}, a_{4}-a_{5}\right)$.

Now we have to check whether $P$ is invertible in $\mathcal{S}_{2, \infty}(2)$ (we are interested in two sided inverse so we use the fact that $\mathcal{S}_{2, \infty}(2)$ is commutative). Let $Q=$ $z_{1} f_{1}+z_{2} f_{2}$. Then $P Q=Q P=\left(x z_{1}\right) f_{1}+\left(y z_{1}+x z_{2}+\mu y z_{2}\right) f_{2}$. Thus $Q$ is the inverse of $P$ iff $x z_{1}=1$ and $y z_{1}+(x+\mu y) z_{2}=1$. Thus $P$ is invertible iff $x \neq 0$ and $x+\mu y \neq 0$. If $P$ is invertible then $P^{-1}=\frac{1}{x} f_{1}+\frac{x-y}{(x+\mu y) x} f_{2}$. From the above it follows that $L$ is in $D(3)$ iff $a_{4}=a_{5}$ or $\left(a_{2}+\mu a_{5}\right)\left(a_{2}+\mu a_{4}\right) \neq 0$. Thus $D(3)$ contains the complement of an algebraic set so it is dense in $\mathcal{S}_{2, \infty}(3)$.

THEOREM 3.4.

(a) Choose any tangles $L \in \mathcal{S}_{2, \infty}(3)$ and $T_{i} \in \mathcal{S}_{2, \infty}(2)$. Further choose any cyclic word, $w\left(L, T_{i}\right)$, over the alphabet $\left\{L, T_{i}\right\}$ and place the corresponding tangles in the annulus as in Fig. 3.12(a). Now consider the cyclic word $w\left(r_{z}(L), T_{i}\right)$, and again place the corresponding tangles in the annulus (Fig. 3.12(b)). Then the elements of the Kauffman bracket skein module of the annulus corresponding to $w\left(L, T_{i}\right)$ and $w\left(r_{z}(L), T_{i}\right)$ are the same.

(b) Choose any tangles $L \in \mathcal{S}_{2, \infty}(3)$ and tangles $T_{i} \in \mathcal{S}_{2, \infty}(2)$. Choose any cyclic word, $w\left(L, T_{i}\right)$, over the alphabet $\left\{L, T_{i}\right\}$ and place the corresponding tangles in the annulus as in Fig. 3.13(a). Now consider the cyclic word 

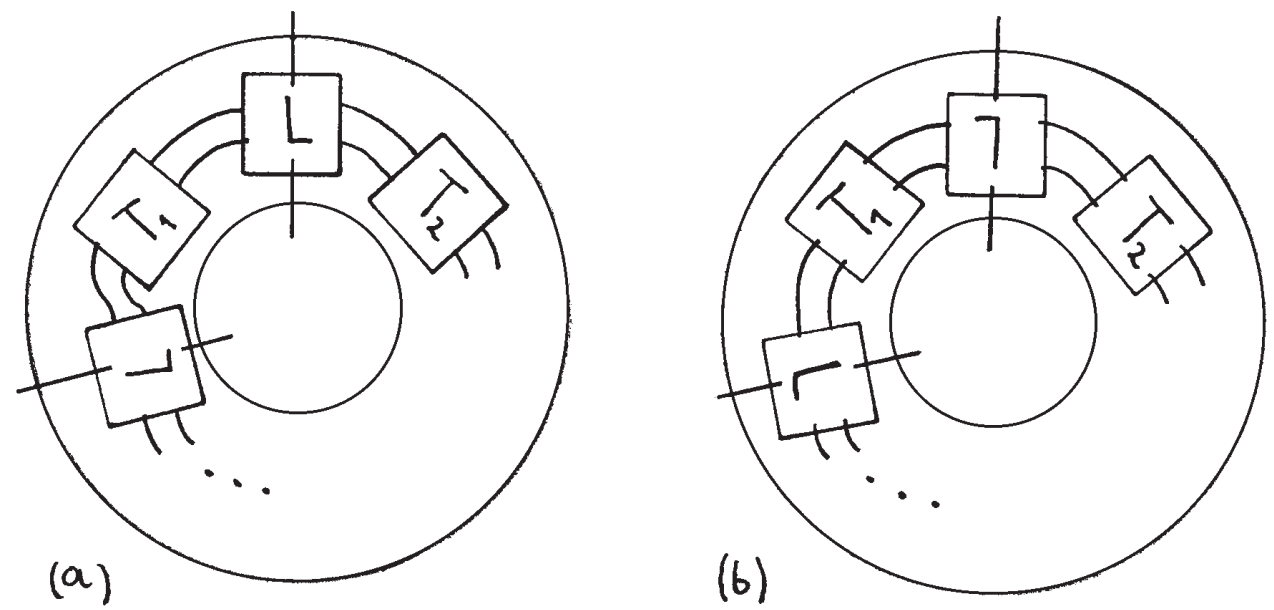

Fig. 3.12
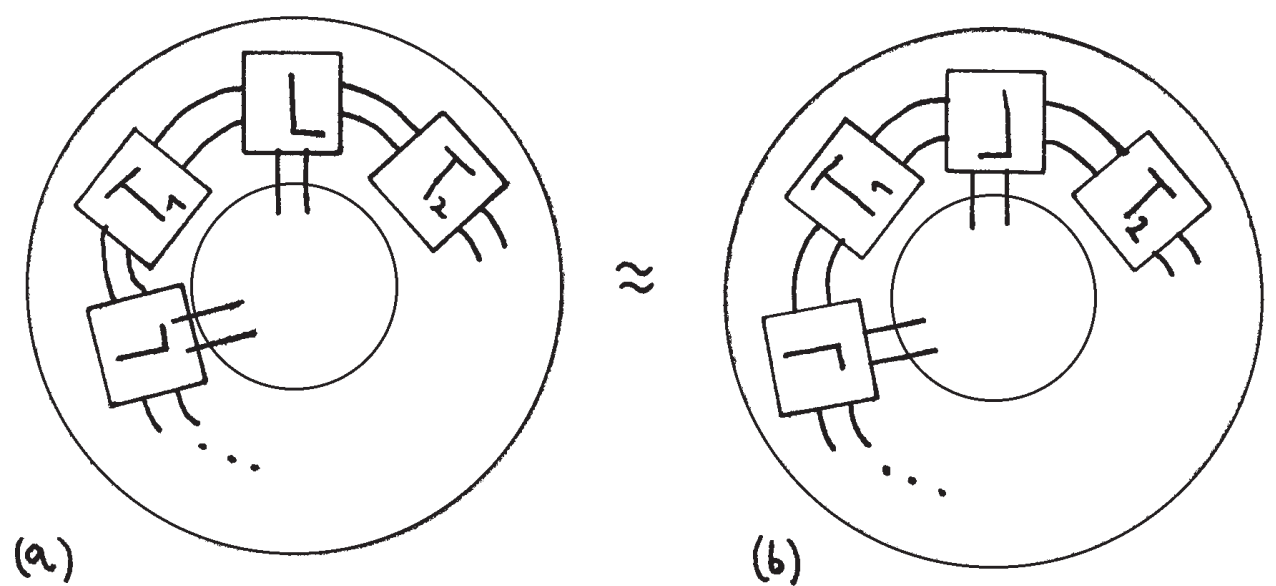

Fig. 3.13

$w\left(r_{y}(L), T_{i}\right)$, and again place the corresponding tangles in the annulus (Fig. 3.13(b)). Then the elements of the skein module of the annulus corresponding to $w\left(L, T_{i}\right)$ and $w\left(r_{y}(L), T_{i}\right)$ are the same.

Proof. We will prove (b). The proof of (a) is analogous ${ }^{5}$. Let $L \in D(3)$ and $P \in \mathcal{S}_{2, \infty}(2)$ from Lemma 3.3(b). Let us place $P P^{-1}$ in the annulus, as in Fig. 3.14. Then let $P$ travel along the annulus, changing $L$ to $r_{y}(L)$, and finally cancelling $P$ with $P^{-1}$; thus Theorem 3.4 holds for a dense subset of $\mathcal{S}_{2, \infty}(3)$ and therefore for any element $L$ of $\mathcal{S}_{2, \infty}(3)$. This completes the proof of Theorem 3.4.

$\overline{5}$ As in [10] , we can deduce (a) from (b). 


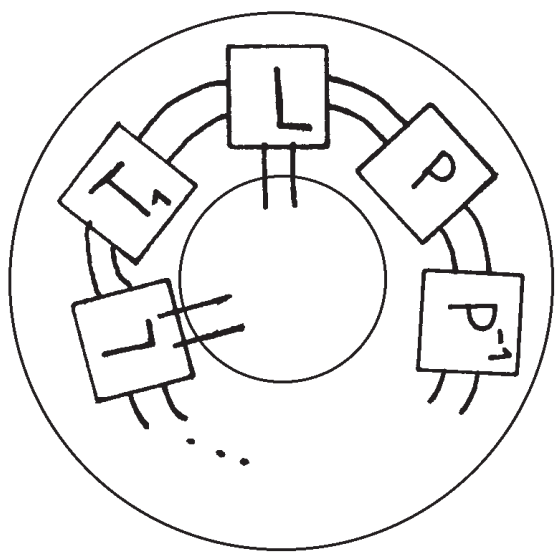

Fig. 3.14

4. Spectral parameter tangle for the skein polynomial. We describe in this section various applications of the Jones idea of the spectral parameter tangle to oriented links, generalizing results of Jones [15] and Traczyk [39]. We can allow various 2 -tangles and variuos mutations, for our construction. There are two essentially different ways of orienting a 3-tangle: "braid like" (Fig. 4.1(a)) and "alternating" (Fig. 4.1(b)). We concentrate here on the braid like case because Traczyk's method does not work in that case.

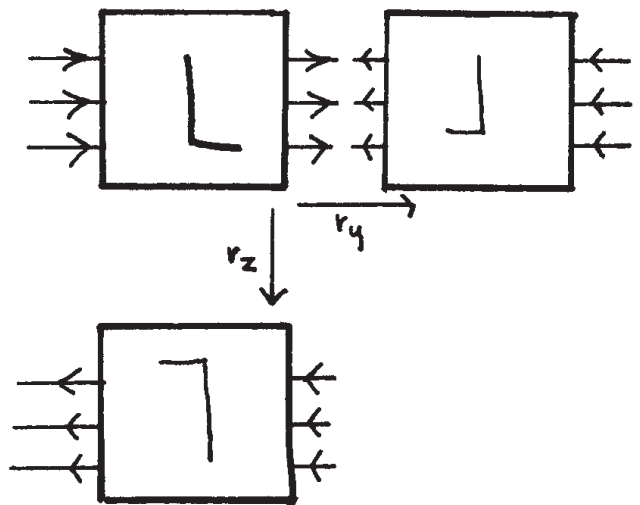

(a)

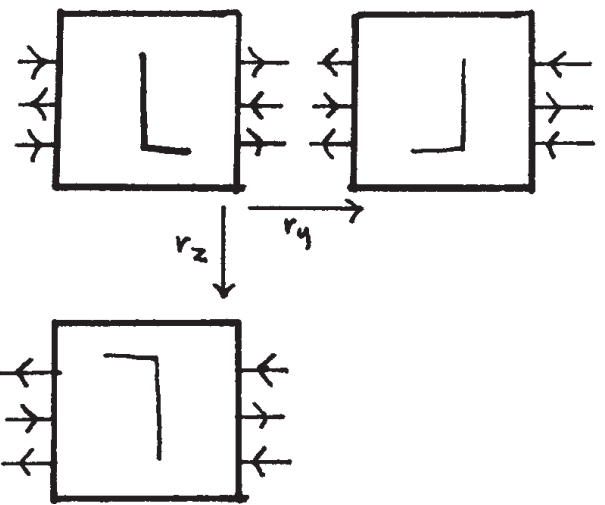

(6)

Fig. 4.1

Remind that the skein (Homflypt) polynomial invariant of oriented links in $S^{3}$, $P_{L} \in Z\left[v^{ \pm 1}, z^{ \pm 1}\right]$, is given by:

(i) $P_{T_{1}}=1$;

(ii) $v^{-1} P_{L_{+}}-v P_{L_{-}}=z P_{L_{0}}$. 
The skein module (linear skein), $\mathcal{S}_{3}(M)$ is a generalization of the skein polynomial to any oriented 3-manifold (possibly with boundary). It is a quotient of $R \mathcal{L}$ and the submodule generated by expressions $v^{-1} P_{L_{+}}-v P_{L_{-}}-z P_{L_{0}}$, where $\mathcal{L}$ is the set of all oriented links (including links with boundary on $\partial M$ ) up to the ambient isotopy, $R$ is a commutative ring with unit containing $Z\left[v^{ \pm 1}, z\right]$ and $R X$ is the free $R$-module with basis $X$. Unless otherwise stated, we work here with $R$ being the field of rational functions, $\mathcal{F}(v, z)$.

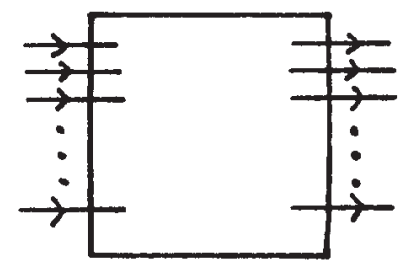

Fig. 4.2

Let $\mathcal{S}_{3}(n)$ denote the skein module of a tangle with $n$ inputs and $n$ outputs as in Fig. 4.2 (inputs and outputs are fixed).
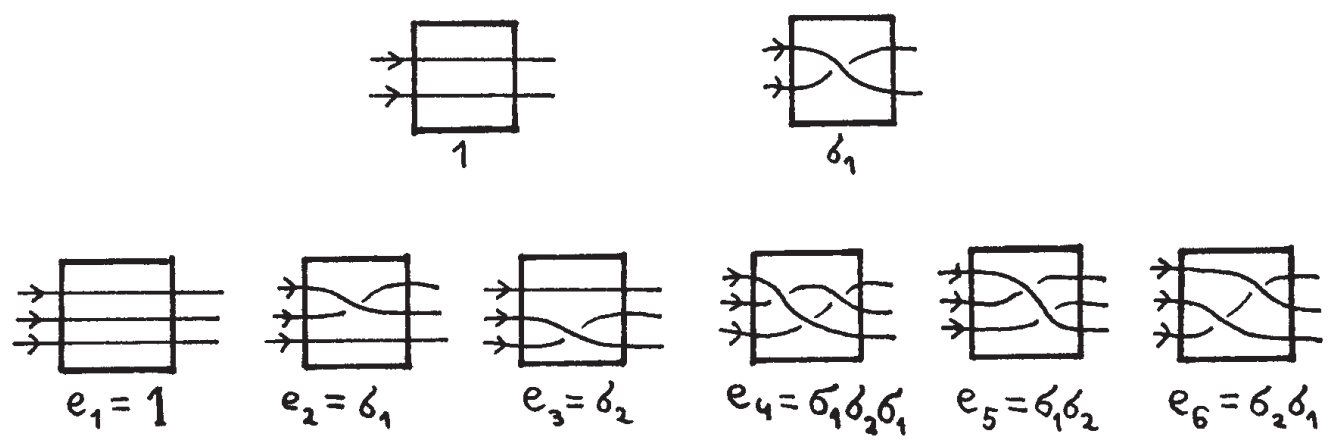

Fig. 4.3

$\mathcal{S}_{3}(n)$ is known to be the Hecke algebra of type $A$ and is free with basis indexed by permutations, $S_{n}[4,12,27]$. In particular $\left(1, \sigma_{1}\right)$ is a basis of $\mathcal{S}_{3}(2)$ and $\left(e_{1}, \ldots, e_{6}\right)=\left(1, \sigma_{1}, \sigma_{2}, \sigma_{1} \sigma_{2} \sigma_{1}, \sigma_{1} \sigma_{2}, \sigma_{2} \sigma_{1}\right)$ is a basis of $\mathcal{S}_{3}(3)$; Fig. 4.3.

LEMMA 4.1.

(a) Let $P=\left(x_{1}+x_{2} \sigma_{1}\right) \in \mathcal{S}_{3}(2) . P$ is invertible in $\mathcal{S}_{3}(2) \quad$ iff $\quad x_{1}^{2}-v^{2} x_{2}^{2}-$ $v z x_{1} x_{2} \neq 0$.

(b) Let $L=\left(\Sigma_{i=1}^{6} a_{i} e_{1}\right) \in \mathcal{S}_{3}(3)$. If $L P=r_{y}(L P)$ up to the global change of orientation, then $x_{1}\left(a_{5}-a_{6}\right)=x_{2}\left(a_{3}+v z a_{6}-v^{2} a_{4}\right)$.

(c) Let $D_{3}(3)$ denote the subset of $\mathcal{S}_{3}(3)$ such that for $L \in D_{3}(3)$, there is an invertible element $P \in \mathcal{S}_{3}(2)$ satisfying $L P=r_{y}(L P)$ (up to the global change of orientation) in $\mathcal{S}_{3}(3)$ (see Fig. 4.4). Then $L \in D_{3}(3)$ iff $a_{5}=a_{6}$ 

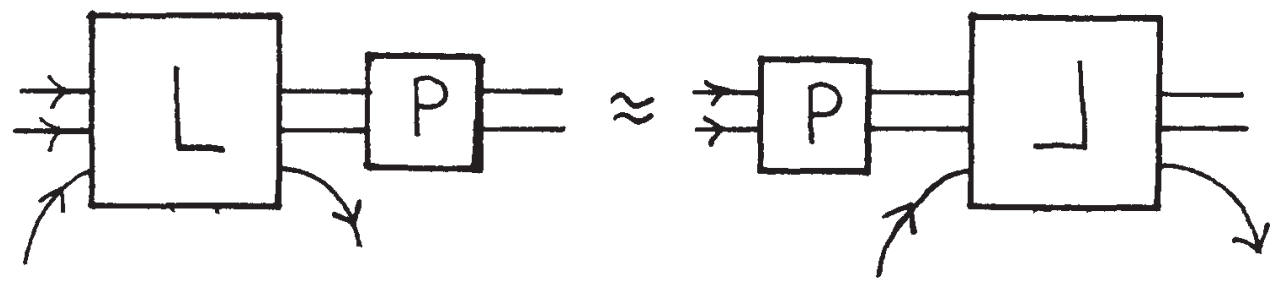

Fig. 4.4

or $F\left(a_{3}, a_{4}, a_{5}, a_{6}\right) \neq 0$ where $F\left(a_{3}, a_{4}, a_{5}, a_{6}\right)=\left(a_{3}-v^{2} a_{4}+v z a_{5}\right)\left(a_{3}-\right.$ $\left.v^{2} a_{4}+v z a_{6}\right)-v^{2}\left(a_{5}-a_{6}\right)^{2}$.

(d) $D_{3}(3)$ is dense in $\mathcal{S}_{3}(3)$.

Proof.

(a) $P$ acts from the right side on $\mathcal{S}_{3}(2)$ and in the basis $1, \sigma_{1}$ it is described by the matrix

$$
[P]=\left[\begin{array}{cc}
x_{1} & v^{2} x_{2} \\
x_{2} & x_{1}+v z x_{2}
\end{array}\right]
$$

Thus $P$ is invertible iff $\operatorname{det}[P]=x_{1}^{2}-v^{2} x_{2}^{2}-v z x_{1} x_{2} \neq 0$.

(b) Let $\langle-,-\rangle$ be a bilinear form on $\mathcal{S}_{3}(3)$ given by $\left\langle e_{i}, e_{j}\right\rangle=\delta_{i, j}$, then $L P=$ $r_{y}(L P)$ is equivalent to $\left\langle L P, e_{5}\right\rangle=\left\langle L P, e_{6}\right\rangle$. The short calculation gives: $\left\langle L P, e_{5}\right\rangle=x_{1} a_{5}+x_{2} a_{4} v^{2}, \quad$ and $\left\langle L P, e_{6}\right\rangle=x_{1} a_{6}+a_{3} x_{2}+v z a_{6} x_{2}$. Thus $P$ is given by the condition $x_{1}\left(a_{5}-a_{6}\right)=x_{2}\left(a_{3}+v z a_{6}-v^{2} a_{4}\right)$.

(c) If $a_{5}=a_{6}$ then we can take for example $P=P^{-1}=1$, otherwise we have unique "projective" solution $P=t\left(\left(a_{3}+v z a_{6}-v^{2} a_{4}\right)+\left(a_{5}-a_{6}\right) \sigma_{1}\right)$. Putting this solution to the condition from (a) one gets: $\left(a_{3}-v^{2} a_{4}+v z a_{5}\right)\left(a_{3}-\right.$ $\left.v^{2} a_{4}+v z a_{6}\right)-v^{2}\left(a_{5}-a_{6}\right)^{2} \neq 0$.

(d) It is enough to notice that the last polynomial inequality holds for a (open) dense subset of $\mathcal{S}_{3}(3)$ (a complement of an algebraic set).

THEOREM 4.2.

(a) Let $L$ be any oriented 3-tangle such that the rotation $r_{z}$ sends inputs to outputs and vice versa, as shown in Fig. 4.5(a). Let o $(L)$ denote the 3-tangle obtained from $L$ by changing its orientation, Fig. 4.5(b).

Consider now a cyclic word $w\left(L, o(L), D_{\alpha}\right)$ over the alphabet consisting of tangles $L, o(L)$ and all oriented 2-tangles, $D_{\alpha}$. Furthermore assume that neighboring tangles in the cyclic word have compatible orientation (can be glued together). Let $T(w)$ be the associated tangle placed in the annulus (oriented version of Fig. 3.12(a)). Now, let us rotate, along the axis $z$, each 3-tangle of the word, and then change its orientation. We obtain, possibly 


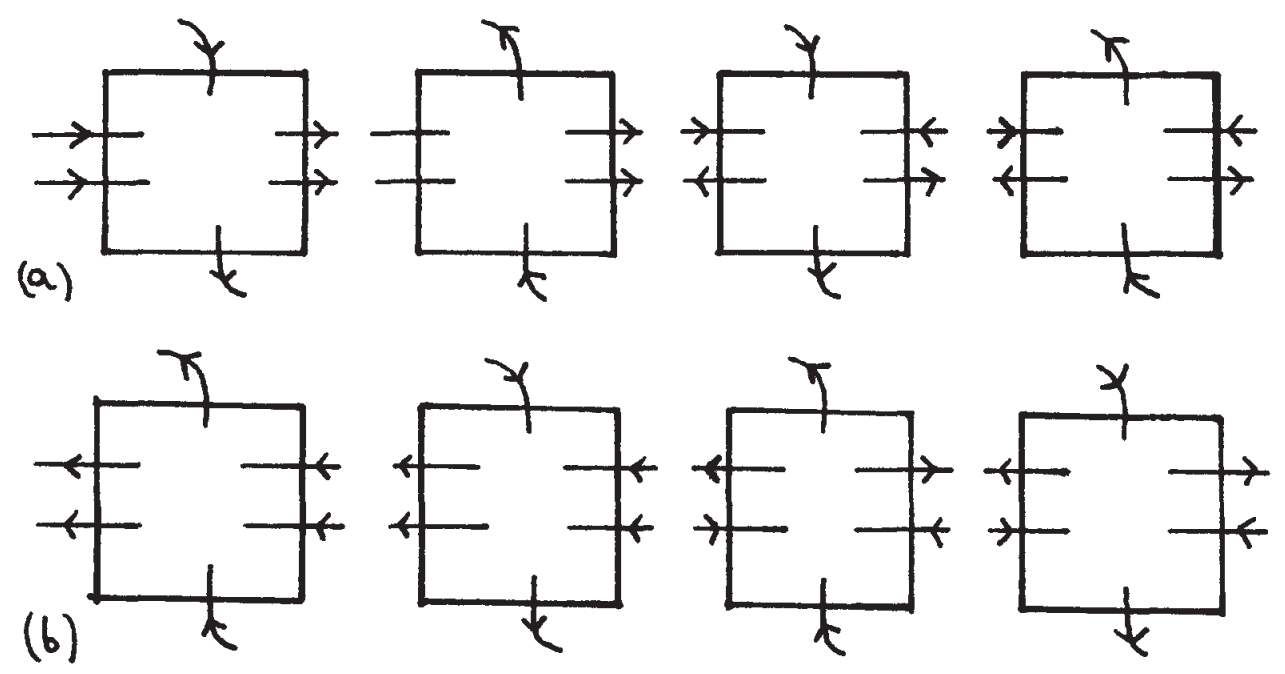

Fig. 4.5

different, tangle but equal to the previous one in the skein module of the solid torus. That is, $T\left(w\left(L, o(L), D_{\alpha}\right)\right)$ and $T\left(w\left(o\left(r_{z}(L)\right), o\left(r_{z}(o(L))\right), D_{\alpha}\right)\right)$ are equal in the skein module of the solid torus; compare Fig. 3.12. If the solid torus is embedded in a 3-manifold and the endpoints of the tangles are connected in the same manner, outside the solid torus, then the resulting links are equal in the skein module of the 3-manifold.

(b) Let $L$ be any oriented 3-tangle such that the rotation $r_{y}$ sends inputs to outputs and vice versa, as shown in Fig. 4.6. Consider now a cyclic word $w\left(L, o(L), D_{\alpha}\right)$ over the alphabet consisting of tangles $L, o(L)$ and all oriented 2-tangles, $D_{\alpha}$. Furthermore assume that neighboring tangles in the cyclic word have compatible orientation (can be glued together). Let $T(w)$ be the associated tangle placed in the annulus. Now, let us rotate, along the axis $y$, each 3-tangle of the word, and then change its orientation. We obtain, possibly different, tangle but equal to the previous one in the skein module of the solid torus. That is, $T\left(w\left(L, o(L), D_{\alpha}\right)\right)$ and $T\left(w\left(o\left(r_{y}(L)\right), o\left(r_{y}(o(L)), D_{\alpha}\right)\right)\right)$ are equal in the skein module of the solid torus; compare Fig. 3.13. If the solid torus is embedded in a 3-manifold and the endpoints of the tangles are connected in the same manner, outside the solid torus, then the resulting links are equal in the skein module of the 3-manifold.

Proof. If $L$ is a "braid like" 3-tangle (Fig. 4.1(a)), we use the spectral parameter tangle (from Lemma 4.1) similarly as in the case of the Kauffman bracket skein relation. All cases of the orientation of $L$ from Fig. 4.5 and 4.6 easily reduce to the basic cases of "braid like" and "alternating" 3-tangles. For an "alterna- 

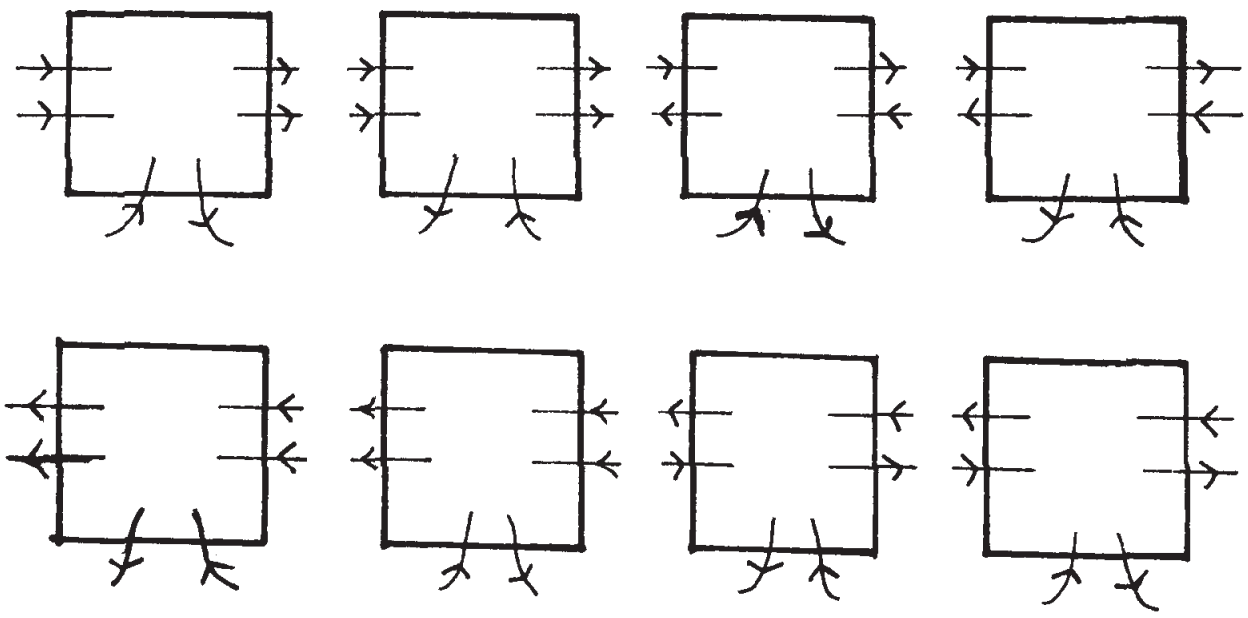

Fig. 4.6

ting" 3-tangles one should prove a lemma analogous to Lemma 4.1, but there is no difficulty in doing so.

5. Spectral parameter 3-tangle. We use in this section the idea of the spectral parameter tangle but in the more involved case. The spectral parameter tangle is, in this section, a 3 -tangle. We work in this part with the third (Homflypt) skein relation, i.e. with the skein module $\mathcal{S}_{3}$. For the Kauffman bracket skein module, computations are similar but slightly shorter. I delayed publishing these results, which were ready in the summer of 1991 [32], because I believed that a similar result could hold for the Kauffman polynomial. Only two years later Traczyk performed calculations which showed that it is not a case (even for a 2cable of the Jones polynomial which gives a special substitution of the Kauffman polynomial).

Theorem 5.1. If $X$ and $Y$ are 3-tangles oriented as in Figure 5.1(a) (resp. Fig. 5.1(b)) and $W(X Y)$ is any word in letters $X$ and $Y$ then

$$
\operatorname{Tr}(W(X, Y))=\operatorname{Tr}\left(W\left(r_{y}(X), r_{y}(Y)\right)\right.
$$

where $\operatorname{Tr}(A)$ denotes the cyclic closer of the 6-tangle $A$ (i.e. the link diagram in the annulus determined by $A$ ); compare Fig. 5.2.

Theorem 5.1 contains 2 parts depending on whether tangles $X$ and $Y$ are of type (a) or (b) of Figure 5.1. The idea in both cases is the same but specific calculations are different. We prove here the simpler case of tangles $X, Y$ oriented as in Figure 5.1(a) ${ }^{6}$. As in previous parts the crucial element of the proof is the

\footnotetext{
6 The initial proof of the second case with segments oriented as in Figure 5.1(b) was performed with the using the computer program Mathematica (and with help of J. Walsh). We will present a "computer free" proof in the future paper [33].
} 


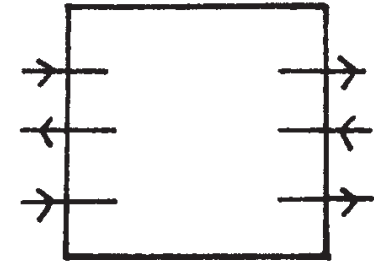

(a)

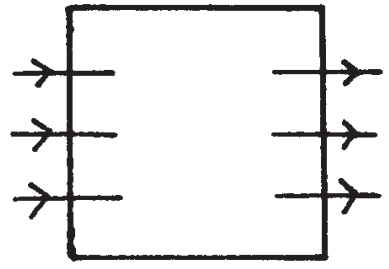

(6)

Fig. 5.1
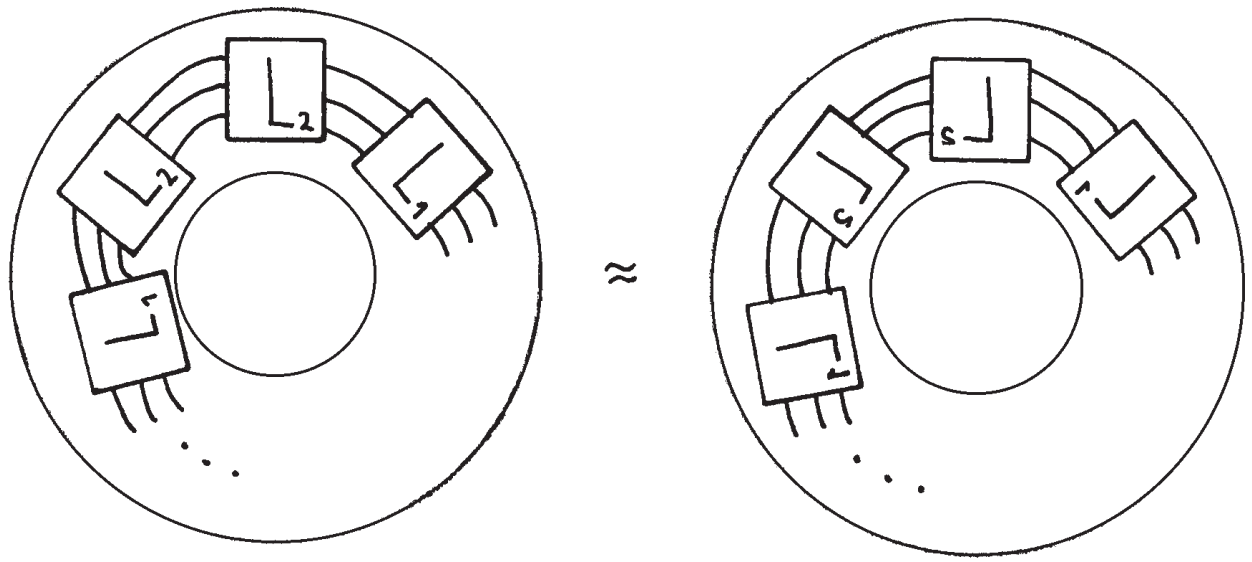

Fig. 5.2

existence of the invertible spectral parameter tangle and then Theorem 5.1 follows easily, as the results in the previous parts. Denote by $\mathcal{S}_{3}^{\prime}(3)$ the skein module of the tangle from Fig. 5.1(a).

LEMMA 5.2.

(a) Let $Y=\Sigma_{i=0}^{5} y_{i} e_{i} \in \mathcal{S}_{3}^{\prime}(3)$, in the basis of $\mathcal{S}_{3}^{\prime}(3)$ shown in Fig. 5.3. $Y$ is invertible in $\mathcal{S}_{3}^{\prime}(3)$ iff

$$
\begin{aligned}
\left(\left(y_{0}+y_{3}+\mu y_{1}\right)\left(y_{0}+y_{4}+\mu y_{2}\right)\right. & \left.-\left(y_{2}+y_{5}+\mu y_{4}\right)\left(y_{1}+y_{5}+\mu y_{3}\right)\right) \\
& \times\left(y_{0}\left(y_{0}+v^{-1} z y_{5}\right)-v^{-2} y_{5}^{2}\right) \neq 0 .
\end{aligned}
$$

(b) Let $X=\Sigma_{i=0}^{5} x_{i} e_{i} \in \mathcal{S}_{3}^{\prime}(3)$. If $X Y=r_{y}(X Y)$ and $Y=r_{y}(Y)$ (up to the global change of orientation) then $y_{3}=y_{4}$ and

$$
\begin{aligned}
y_{0}\left(x_{4}-x_{3}\right) & +y_{1}\left(x_{2}+x_{5}+\mu x_{4}\right)-y_{2}\left(x_{1}+x_{5} \mu x_{3}\right) \\
& -y_{3}\left(x_{3}-x_{4}+\mu\left(x_{1}-x_{2}\right)\right)+y_{5}\left(x_{2}-x_{1}\right)=0 .
\end{aligned}
$$

(c) Let $D^{2}(3)$ denote the subset of $\mathcal{S}_{3}^{\prime}(3) \times \mathcal{S}_{3}^{\prime}(3)$, such that $(A, B) \in D^{2}(3)$ iff there is an invertible element $Y \in \mathcal{S}_{3}^{\prime}(3)$ such that $A Y=Y r_{y}(A)$ and $B Y=Y r_{y}(B)$. Then $D^{2}(3)$ is dense in $\mathcal{S}_{3}^{\prime}(3) \times \mathcal{S}_{3}^{\prime}(3)$. 


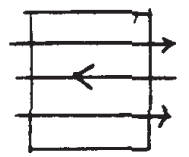

$e_{0}$

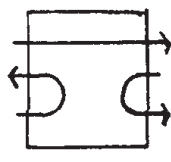

$e_{1}$

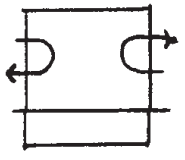

$e_{2}$

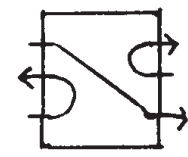

$e_{3}$

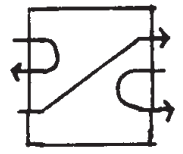

$e_{4}$

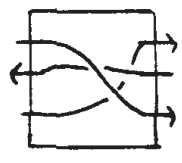

$e_{5}$

Fig. 5.3

Proof.

(a) Let $X=\Sigma_{i=0}^{5} x_{i} e_{i}, Y=\Sigma_{i=0}^{5} y_{i} e_{i}$, in the basis of $\mathcal{S}_{3}^{\prime}(3)$ shown in Fig. 5.3.

Then

$$
\begin{aligned}
& X Y=e_{0}\left(y_{0} x_{0}+v^{-2} y_{5} x_{5}\right) \\
& +e_{1}\left(y_{0} x_{1}+y_{1}\left(x_{0}+x_{3}+\mu x_{1}\right)+y_{4}\left(x_{1}+x_{5}+\mu x_{3}\right)+y_{5}\left(x_{3}-v^{-1} z x_{5}\right)\right) \\
& +e_{2}\left(y_{0} x_{2}+y_{2}\left(x_{0}+x_{4}+\mu x_{2}\right)+y_{3}\left(x_{2}+x_{5}+\mu x_{4}\right)+y_{5}\left(x_{4}-v^{-1} z x_{5}\right)\right) \\
& +e_{3}\left(y_{0} x_{3}+y_{2}\left(x_{1}+x_{5}+\mu x_{3}\right)+y_{3}\left(x_{0}+x_{3}+\mu x_{1}\right)+y_{5} x_{1}\right) \\
& +e_{4}\left(y_{0} x_{4}+y_{1}\left(x_{2}+x_{5}+\mu x_{4}\right)+y_{4}\left(x_{0}+x_{4}+\mu x_{2}\right)+y_{5} x_{2}\right) \\
& +e_{5}\left(y_{0} x_{5}+y_{5}\left(x_{0}+v^{-1} z x_{5}\right)\right) .
\end{aligned}
$$

If $X$ is treated as a linear operator acting (from the left) on $\mathcal{S}_{3}^{\prime}(3)$, then from the above calculation we obtain that in the basis $e_{1}, \ldots, e_{5}$ :

$$
\begin{aligned}
\operatorname{det} X & =\left(\left(x_{0}+x_{3}+\mu x_{1}\right)\left(x_{0}+x_{4}+\mu x_{2}\right)\right. \\
& \left.-\left(x_{2}+x_{5}+\mu x_{4}\right)\left(x_{1}+x_{5}+\mu x_{3}\right)\right)^{2}\left(x_{0}\left(x_{0}+v^{-1} z x_{5}\right)-v^{-2} x_{5}^{2}\right) .
\end{aligned}
$$

Then part (a) follows (we have to change roles of $X$ and $Y$ ).

(b) follows immediately from the formula for $X Y$.

(c) One have to perform easy but long and tedious calculations.

One of the ingredients of our work was to show that the elements of the Temperley-Lieb algebra $T L_{2}$ and $T L_{3}$ are almost everywhere invertible. We can show that it holds for any $n$, answering the question raised by Rolfsen in [36] (Question after Proposition 5). Similar fact also holds for the Hecke algebra $H_{n}(v, z)=\mathcal{S}_{3}(n)$ as well as for Birman-Murakami-Wenzl algebra.

THEOREM 5.3.

(a) Invertible elements of $T L_{n}=\mathcal{S}_{2, \infty}(n)$ form a dense subset of $T L_{n}$.

(b) Invertible elements of $H_{n}(v, z)$ form a dense subset of $H_{n}(v, z)$.

Proof. We will demonstrate (b). The proof of (a) is similar. The main idea of the proof is observation that the analogous result for the group algebra over the symmetric group $S_{n}$ can be seen immediately ${ }^{7}$.

\footnotetext{
7 We could stop here saying that $H_{n}(v, z)$ and $\mathcal{F}(v, z) S_{n}$ are algebra isomorphic, but we present an elementary proof.
} 
It is convenient to work with the $\operatorname{ring} Q\left[v^{ \pm 1}, z\right]$, in addition to the field $\mathcal{F}(v, z)$. Denote the corresponding (to the ring $Q\left[v^{ \pm 1}, z\right]$ ) skein module and Hecke algebra by $\mathcal{S}_{3}^{+}(n)=H_{n}^{+}(v, z)$. Let $\left\{e_{1}=1, e_{2}, \ldots, e_{n !}\right\}$ be a basis of $H_{n}(v, z)$, composed of positive braids of minimal number of crossings for a given permutation. It is also a basis of the free module $H_{n}^{+}(v, z)$. Let $X$ be an element of $H_{n}(v, z)$ and consider its action from the left on $H_{n}(v, z)$. It is a linear function and its matrix $[X]=\left[a_{i, j}\right]$ in our basis is given by $X e_{i}=\Sigma_{j} a_{i, j} e_{j}$. Invertible elements of $H_{n}(v, z)$ form a subalgebra of $H_{n}(v, z)$. It is immediate but important observation that in order to show that they are dense in $H_{n}(v, z)$, it suffices to show that elements of non-zero determinant in $H_{n}^{+}(v, z)$ are dense in $H_{n}^{+}(v, z)$. Consider $X=\sum_{i=1}^{n !} x_{i} e_{i}$ in $H_{n}^{+}(v, z)$. Because $x_{i} \in Q\left[v^{ \pm 1}, z\right]$, we can work now modulo $\left(z, v^{2}-1\right)$ - the ideal generated by $z$ and $v^{2}-1$. Our skein relation $\sigma_{i}^{2}=v z \sigma_{i}+v^{2}$ reduces to $\sigma_{i}^{2} \equiv 1 \bmod \left(z, v^{2}-1\right)$. We can immediately check that $\operatorname{det} X \equiv$ $x_{1}^{n !}+\mathcal{O}\left(x_{1}^{n !-2}\right) \bmod \left(z, v^{2}-1\right)$, because for $[X]=\left[a_{i, j}\right], a_{i, i} \equiv x_{1} \bmod \left(z, v^{2}-1\right)$ and $a_{i, j} \equiv x_{s} \bmod \left(z, v^{2}-1\right)$ where $s \neq 1$ for $i \neq j$. Thus $\operatorname{det} X \neq 0$ outside an algebraic set and Theorem 5.3(b) holds.

Another application of the spectral parameter tangle has been recently demonstrated by Rolfsen in [37]. Furthermore, the remarkable example by T. Kanenobu of an infinite family of different knots with the same skein polynomial [17] can be put in more general context similar to that of the spectral parameter tangle [18]. Namely Kanenobu considers elements of $\mathcal{S}_{3}^{\prime}(n)$ for which $e_{2}$ of Figure 5.3 is an eigenvector.

\section{References}

[1] D. Albers, John Horton Conway, Talking a good game, Math. Horizons, Spring 1994, Published by the M.A.A.

[2] R. P. Anstee, J. H. Przytycki, D. Rolfsen, Knot polynomials and generalized mutation, Topology Appl., 32 (1989), 237-249.

[3] R. Baxter, Exactly solved models in statistical mechanics, Academic Press, London, 1982.

[4] N. Bourbaki, Groupes et algèbres de Lie, VI: Groupes de Coxeter et systèmes de Tits, Herman Paris, 1968

[5] R. I. Brooks, C. A. B. Smith, A. H. Stone, W. T. Tutte, The dissection of rectangles into squares, Duke Math. J. 7 (1940), 312-340.

[6] J. H. Conway, An enumeration of knots and links, Computational problems in abstract algebra (ed. J. Leech), Pergamon Press (1969), 329-358.

[7] P. Freyd, D. Yetter, J. Hoste, W. B. R. Lickorish, K. Millett, A. Ocneanu, A new polynomial invariant of knots and links, Bull. Amer. Math. Soc. 12 (1985), 239-249.

[8] J. Hoste, A polynomial invariant of knots and links, Pacific J. Math. 124 (1986), 295-320.

[9] J. Hoste, J. H. Przytycki, A survey of skein modules of 3-manifolds, in: Knots 90, De Gruyter, Berlin - New York 1992, 363-379. 
[10] J. Hoste, J. H. Przytycki, Tangle surgeries which preserve Jones-type polynomials, Center for Pure and Applied Mathematics preprint - PAM 617, U. C. Berkeley, 1994.

[11] G. T. Jin, D. Rolfsen, Some remarks on rotors in link theory, Canad. Math. Bull. 34 (1991), 480-484.

[12] V. Jones, Hecke algebra representations of braid groups and link polynomials, Ann. of Math. 126 (1987), 335-388.

[13] V. F. R. Jones, On knot invariants related to some statistical mechanical models, Pacific J. Math. 137 (1989), 311-334.

[14] V. F. R. Jones, Talk given at 25th Annual Spring Topology Conference, CSU Sacramento, April 11, 1991.

[15] V. F. R. Jones, Commuting transfer matrices and link polynomials, Internat. J. Math. 3 (1992), 205-212.

[16] V.F. R. Jones, Coincident link polynomials from commuting transfer matrices, Proceedings of the XXth International Conference on Differential Geometric Methods in Theoretical Physics, Vol. 1, 2 (New York, 1991), 137-151, World Sci. Publishing, River Edge, NJ, 1992.

[17] T. Kanenobu, Infinitely many knots with the same polynomial invariant, Proc. Amer. Math. Soc. 97 (1986), 158-162.

[18] T. Kanenobu, The Homfly and the Kauffman bracket polynomials for the generalized mutant of a link, Topology Appl., to appear.

[19] J. Kania-Bartoszyńska, Examples of different 3-manifolds with the same invariants of Witten and Reshetikhin-Turaev, Topology 32 (1993), 47-54.

[20] L. H. Kauffman, State models and the Jones polynomial, Topology 26 (1987), 395-407.

[21] W. B. R. Lickorish, Polynomials for links, Bull. London Math. Soc. 20 (1988), 558-588.

[22] W. B. R. Lickorish, Distinct 3-manifolds with all $S U(2)_{q}$ invariants the same, Proc. Amer. Math. Soc. 117 (1993), 285-292.

[23] W. B. R. Lickorish, A. S. Lipson, Polynomials of 2-cable-like links, Proc. Amer. Math. Soc. 100 (1987), 355-361.

[24] W. B. R. Lickorish, K. Millett, A polynomial invariant of oriented links, Topology 26 (1987), 107-141.

[25] J. M. Montesinos, Surgery on links and double branched covers of $S^{3}$, in: Knots, groups and 3-manifolds, ed. L. P. Neuwirth, Ann. Math. Studies, 84, 227-259, Princeton Univ. Press, 1975.

[26] H. R. Morton, P. Traczyk, The Jones polynomial of satellite links around mutants, in: Braids, Ed. J. S. Birman, A. Libgober, AMS Contemporary Math., 78 (1988), 587-592.

[27] H. R. Morton, P. Traczyk, Knots and algebras, Contribuciones Matematicas en homenaje al profesor D. Antonio Plans Sanz de Bremond, ed. E. Martin-Peinador and A. Rodez Usan, University of Zaragoza, (1990), 201-220.

[28] J. Murakami, The parallel version of polynomial invariants of links, Osaka J. Math. 26 (1989), $1-55$.

[29] J. H. Przytycki, Equivalence of cables of mutants of knots, Canad. J. Math. XLI (1989), $250-273$. 
[30] J. H. Przytycki, Skein modules of 3-manifolds, Bull. Polish Acad. Sci. Math. 39 (1991), $91-100$.

[31] J. H. Przytycki, Manuscript of the lecture delivered at the University of Tennessee, October 18, 1991.

[32] J. H. Przytycki, Applications of the spectral parameter tangle of $V$. Jones, Abstracts Amer. Math. Soc. 12 (1991), 496-497.

[33] J. H. Przytycki, The spectral parameter 3-string tangle, in preparation.

[34] J. H. Przytycki, P. Traczyk, Invariants of links of Conway type, Kobe J. Math. 4 (1987), $115-139$.

[35] N. Y. Reshetikhin, V. Turaev, Invariants of three manifolds via link polynomials and quantum groups. Invent. Math. 103 (1991), 547-597.

[36] D. Rolfsen, The quest for a knot with trivial Jones polynomial; diagram surgery and the Temperley-Lieb algebra, in: Topics in knot theory, Ed. M. E. Bozhüyük, NATO ASI Series, Series C: Mathematical and Physical Sciences - Vol. 399, Kluwer Academic Publishers 1993, 195-210.

[37] D. Rolfsen, Global mutation of knots, J. Knot Theory Ramifications 3 (1994), 407-417.

[38] H. N. V. Temperley, E. H. Lieb, Relations between the "percolation" and "coloring" problem and other graph-theoretical problems associated with regular planar lattices: some exact results for the "percolation" problem, Proc. Roy. Soc. London Ser. A 322 (1971), 251-280.

[39] P. Traczyk, A note on rotant links, preprint, 1989.

[40] V. Turaev, The Yang-Baxter equation and invariants of links, Invent. Math. 92 (1988), $527-553$.

[41] V. G. Turaev, The Conway and Kauffman modules of the solid torus, Zap. Nauchn. Sem. Leningrad. Otdel. Mat. Inst. Steklov. (LOMI) 167 (1988), 79-89.

[42] V. G. Turaev, H. Wenzl, Quantum invariants of 3-manifolds associated with classical simple Lie algebras, Internat. J. Math. 4 (1993), 323-358.

[43] W. T. Tutte, Codichromatic graphs, J. Combin. Theory Ser. B 16 (1974), 168-174.

[44] O. Ya. Viro, Nonprojecting isotopies and knots with homeomorphic coverings, J. Soviet Math. 12 (1979), 86-96.

[45] F. Waldhausen, Über Involutionen der 3 Sphäre, Topology 8 (1969), 81-91.

[46] S. Yamada, An operator on regular isotopy invariants of link diagrams, Topology 28 (1989), 369-377. 